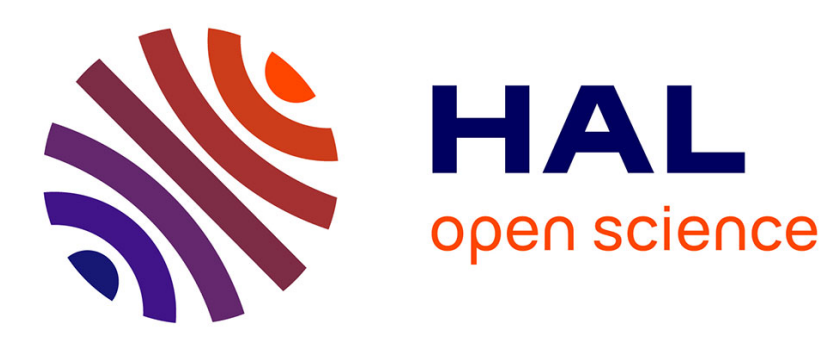

\title{
A dual approach to Kohn-Vogelius regularization applied to data completion problem
}

\author{
Fabien Caubet, Jérémi Dardé
}

\section{To cite this version:}

Fabien Caubet, Jérémi Dardé. A dual approach to Kohn-Vogelius regularization applied to data completion problem. Inverse Problems, 2020, 10.1088/1361-6420/ab7868 . hal-02310869

\section{HAL Id: hal-02310869 \\ https://hal.science/hal-02310869}

Submitted on 10 Oct 2019

HAL is a multi-disciplinary open access archive for the deposit and dissemination of scientific research documents, whether they are published or not. The documents may come from teaching and research institutions in France or abroad, or from public or private research centers.
L'archive ouverte pluridisciplinaire HAL, est destinée au dépôt et à la diffusion de documents scientifiques de niveau recherche, publiés ou non, émanant des établissements d'enseignement et de recherche français ou étrangers, des laboratoires publics ou privés. 


\title{
A dual approach to Kohn-Vogelius regularization applied to data completion problem
}

\author{
Fabien Caubet* and Jérémi Dardé ${ }^{\dagger}$
}

October 10, 2019

\begin{abstract}
This paper focuses on a dual approach in order to study the data completion problem. A classical method to solve this problem is to minimize the so-called regularized Kohn-Vogelius functional. However this method needs to choose an appropriate parameter of regularization to ensure its efficiency in the numerical reconstruction. To avoid this difficulty, we propose to study the inverse problem through a dual problem.

Using some well-chosen functional spaces and establishing theoretical results in a abstract setting, we prove the well-posedness of the dual minimization problem and the convergence of our regularized solution to the exact solution when the amount of noise on the data goes to 0 . Moreover we prove that the regularized solution satisfies the well-known Morozov discrepancy principle. Then we establish that the minimization of the dual functional permits not only to stably obtain a good reconstruction of the missing data of the Cauchy problem but also to determine the value of a suitable parameter of regularization in the Kohn-Vogelius strategy. We finally present numerical results, in two and three dimensions, to underline the efficiency of the proposed method.
\end{abstract}

\section{Introduction}

Data completion problem. We are interested in the regularization of the data completion problem, also known as Cauchy problem, for Laplace's equation. More precisely, let $\Omega$ be a connected bounded open domain of $\mathbb{R}^{d}$, where $d=2$ or $d=3$ is the dimension, with a Lipschitz boundary $\partial \Omega$. We assume $\partial \Omega$ to be divided in two open sets $\Gamma$ and $\Gamma_{\mathrm{c}}=\partial \Omega \mid \bar{\Gamma}$ of strictly positive Lebesgue measure. Let $\boldsymbol{\nu}$ be the unit exterior normal vector to $\Omega$. For a Cauchy data $\left(g_{\mathrm{D}}, g_{\mathrm{N}}\right) \in \mathrm{H}^{1 / 2}(\Gamma) \times \mathrm{H}^{-1 / 2}(\Gamma){ }_{1}^{1}$ our problem of interest reads: find $u \in \mathrm{H}^{1}(\Omega)$ such that

$$
\left\{\begin{aligned}
\Delta u & =0 & & \text { in } \Omega, \\
u & =g_{\mathrm{D}} & & \text { on } \Gamma, \\
\partial_{\nu} u & =g_{\mathrm{N}} & & \text { on } \Gamma,
\end{aligned}\right.
$$

where $\partial_{\nu} u$ is the normal derivative of $u$.

${ }^{*}$ CNRS/Univ. Pau \& Pays Adour/E2S UPPA, Laboratoire de Mathématiques et de leurs Applications de Pau-IPRA, UMR 5142, 64000 Pau, France (fabien.caubet@univ-pau.fr)

${ }^{\dagger}$ Institut de Mathématiques de Toulouse UMR5219, Université de Toulouse, CNRS, UPS, F-31062 Toulouse Cedex 9 , France (Jeremi.Darde@math.univ-toulouse.fr)

${ }^{1}$ The functional setting is specified in Appendix A 
It is well known that such problem is severely ill-posed: it admits at most one solution, but fails to have one for a subset of Cauchy data dense in $\mathrm{H}^{1 / 2}(\Gamma) \times \mathrm{H}^{-1 / 2}(\Gamma)$, and presents exponential instabilities with respect to noise (see, e.g., 8, 9, 32]).

From a reconstruction point of view, these instabilities are the main issue: in particular, for any $\varepsilon>0$ and for any data $\left(g_{\mathrm{D}}, g_{\mathrm{N}}\right)$ for which Problem (1.1) admits a solution $u$, there exists another data $\left(\tilde{g}_{\mathrm{D}}, \tilde{g}_{\mathrm{N}}\right)$ for which Problem (1.1) also admits a solution $\tilde{u}$, so that at the same time (see, among others, [25, Section 2])

$$
\left\|g_{\mathrm{D}}-\tilde{g}_{\mathrm{D}}\right\|_{\mathrm{H}^{1 / 2}(\Gamma)}+\left\|g_{\mathrm{N}}-\tilde{g}_{\mathrm{N}}\right\|_{\mathrm{H}^{-1 / 2}(\Gamma)} \leqslant \varepsilon \quad \text { and } \quad\|u-\tilde{u}\|_{\mathrm{H}^{1}(\Omega)} \geqslant \frac{1}{\varepsilon} .
$$

As, from a practical point of view, one should always expect noise on real-life data, it is not only necessary to propose a regularization method that reconstruct a good approximation of the searched solution when exact data are at hand, but it is mandatory to provide a strategy to deal with the noise.

The best stability one can expect for this problem is a logarithmic conditional stability as underlined in the following result (see [3, Theorem 1.9]):

Theorem 1.1. Let $M>0$ and $\delta>0$. There exist $C>0$ and $\mu \in(0,1)$ such that for all Cauchy data $\left(g_{\mathrm{D}}, g_{\mathrm{N}}\right) \in \mathrm{H}^{1 / 2}(\Gamma) \times \mathrm{H}^{-1 / 2}(\Gamma)$ verifying

$$
\left\|g_{\mathrm{D}}\right\|_{\mathrm{H}^{1 / 2}(\Gamma)}+\left\|g_{\mathrm{N}}\right\|_{\mathrm{H}^{-1 / 2}(\Gamma)} \leqslant \delta
$$

for all $u \in \mathrm{H}^{1}(\Omega)$ solution of 1.1 with an a-priori bound on the $\mathrm{H}^{1}$-norm

$$
\|u\|_{\mathrm{H}^{1}(\Omega)} \leqslant M,
$$

one has

$$
\|u\|_{\mathrm{L}^{2}(\Omega)} \leqslant(M+\delta) \omega\left(\frac{\delta}{M+\delta}\right)
$$

where $\omega: \mathbb{R}_{+} \rightarrow \mathbb{R}_{+}$satisfies

$$
\omega(t) \leqslant \frac{C}{\ln \left(\frac{1}{t}\right)^{\mu}}, \quad \forall t \in(0,1) .
$$

In other word, one may restore a very weak stability assuming that the solutions we are looking for are a priori bounded by some constant.

Remark 1.2. In the present article, we focus on Laplace's equation for simplicity. But everything we present easily adapts to a general elliptic data completion problem, with Laplace's equation replaced by a general elliptic equation in divergence form

$$
\operatorname{div}(\sigma \nabla u)=0,
$$

where $\sigma \in \mathrm{W}^{1, \infty}(\Omega)$ satisfies the usual ellipticity condition $\sigma \geqslant c>0$ a.e. in $\Omega$, and where the normal derivative is modified accordingly.

Several regularization techniques has been proposed to tackle Problem 1.1. Without being exhaustive, we may mention methods based on surface integral equations [12, 23, Lavrentiev regularization [10, 11, stabilized finite elements methods [15, 17, quasi-reversibility method [13, 18, 26, 35, 36], fading regularization method [24, 27, etc. 
A dual optimization strategy. In our present work, we focus on an optimization strategy which is closely related to the so-called Kohn-Vogelius strategy. More precisely, and in a sense we will make more accurate in the next section, the proposed strategy is dual to the Kohn-Vogelius optimization problem used in 21] to deal with problem 11.1. This dual strategy is closely related to the one developed in [14, in the context of inverse problems and quasi-reversibility method, but with somehow a reverse point of view. It is also closely related to the works [28, 31] in the context of control theory.

Let $\left(g_{\mathrm{D}}, g_{\mathrm{N}}\right) \in \mathrm{H}^{1 / 2}(\Gamma) \times \mathrm{H}^{-1 / 2}(\Gamma)$ be the exact boundary data, in the sense that they correspond to an exact solution $u_{\mathrm{ex}} \in \mathrm{H}^{1}(\Omega)$ to Problem (1.1) that we seek to reconstruct. From a data completion point of view, we aim to reconstruct the missing data $\left(\varphi_{\mathrm{ex}}, \psi_{\mathrm{ex}}\right)=\left(\partial_{\nu} u_{\mathrm{ex} \mid \Gamma_{\mathrm{c}}}, u_{\mathrm{ex} \mid \Gamma_{\mathrm{c}}}\right) \in \mathrm{H}^{-1 / 2}\left(\Gamma_{\mathrm{c}}\right) \times \mathrm{H}^{1 / 2}\left(\Gamma_{\mathrm{c}}\right)$ from the knowledge of $\left(g_{\mathrm{D}}, g_{\mathrm{N}}\right)$.

We define

$$
\boldsymbol{F}=\nabla u_{\mathrm{N}}-\nabla u_{\mathrm{D}} \in \mathbf{L}^{2}(\Omega),
$$

where $u_{\mathrm{N}}$ and $u_{\mathrm{D}}$ belong to $\mathrm{H}^{1}(\Omega)$ and satisfy $\left.\right|^{2}$ respectively

$$
\left\{\begin{array} { r l r l } 
{ \Delta u _ { \mathrm { N } } } & { = 0 } & { } & { \text { in } \Omega , } \\
{ \partial _ { \nu } u _ { \mathrm { N } } } & { = g _ { \mathrm { N } } } & { } & { \text { on } \Gamma , } \\
{ u _ { \mathrm { N } } } & { = 0 } & { } & { \text { on } \Gamma _ { \mathrm { c } } , }
\end{array} \quad \text { and } \quad \left\{\begin{array}{rlrl}
\Delta u_{\mathrm{D}} & =0 & & \text { in } \Omega, \\
u_{\mathrm{D}} & =g_{\mathrm{D}} & & \text { on } \Gamma, \\
\partial_{\nu} u_{\mathrm{D}} & =0 & & \text { on } \Gamma_{\mathrm{c}} .
\end{array}\right.\right.
$$

We suppose that we have at our disposal a noisy version $\left(g_{\mathrm{D}}^{\delta}, g_{\mathrm{N}}^{\delta}\right) \in \mathrm{H}^{1 / 2}(\Gamma) \times \mathrm{H}^{-1 / 2}(\Gamma)$ of the data such that

$$
\left\|g_{\mathrm{D}}^{\delta}-g_{\mathrm{D}}\right\|_{\mathrm{H}^{1 / 2}(\Gamma)}+\left\|g_{\mathrm{N}}^{\delta}-g_{\mathrm{N}}\right\|_{\mathrm{H}^{-1 / 2}(\Gamma)} \leqslant \delta .
$$

We define $u_{\mathrm{D}}^{\delta}, u_{\mathrm{N}}^{\delta}$ and $\boldsymbol{F}^{\delta}$ as $u_{\mathrm{D}}, u_{\mathrm{N}}$ and $\boldsymbol{F}$, simply replacing $g_{\mathrm{D}}$ and $g_{\mathrm{N}}$ by their noisy counterparts $g_{\mathrm{D}}^{\delta}$ and $g_{\mathrm{N}}^{\delta}$ in (1.2). It is not difficult to see that there exists a constant $c>0$, independent of $\delta, g_{\mathrm{D}}$ and $g_{\mathrm{N}}$, such that

$$
\left\|\boldsymbol{F}^{\delta}-\boldsymbol{F}\right\|_{\mathbf{L}^{2}(\Omega)} \leqslant c \delta .
$$

We also make the classical assumption that $c \delta<\left\|\boldsymbol{F}^{\delta}\right\|_{\mathbf{L}^{2}(\Omega)}$, that is we suppose that the ratio information versus noise is sufficient so that we may hope to reconstruct something.

Remark 1.3. To apply the method we will introduce below, we need to know the constant c, or at least to obtain a good numerical approximation of it. We come back on that matter in Section 5 .

We define

$$
\mathrm{H}_{\diamond}^{-1 / 2}(\partial \Omega)=\left\{\theta \in \mathrm{H}^{-1 / 2}(\partial \Omega),\langle\theta, 1\rangle=0\right\},
$$

and

$$
\mathscr{F}: \theta \in \mathrm{H}_{\diamond}^{-1 / 2}(\partial \Omega) \longmapsto \frac{1}{2} \int_{\Omega}\left(\left|\nabla v_{1}(\theta)\right|^{2}+\left|\nabla v_{2}(\theta)\right|^{2}\right) \mathrm{d} x+c \delta\left(\int_{\Omega}|\nabla w(\theta)|^{2} \mathrm{~d} x\right)^{\frac{1}{2}}-\int_{\Omega} \boldsymbol{F}^{\delta} \cdot \nabla w(\theta) \mathrm{d} x,
$$

where $w(\theta) \in \mathrm{H}^{1}(\Omega)$ verifies $\int_{\Gamma_{\mathrm{c}}} w(\theta) \mathrm{d} s=0$ and

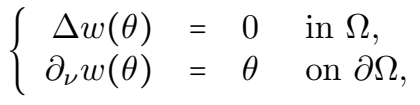

and $v_{1}(\theta)$ and $v_{2}(\theta)$ belong to $\mathrm{H}^{1}(\Omega)$ and verify respectively

$$
\left\{\begin{array} { r l r l } 
{ \Delta v _ { 1 } ( \theta ) } & { = 0 } & { } & { \text { in } \Omega , } \\
{ v _ { 1 } ( \theta ) } & { = 0 } & { } & { \text { on } \Gamma , } \\
{ \partial _ { \nu } v _ { 1 } ( \theta ) } & { = \theta } & { } & { \text { on } \Gamma _ { \mathrm { c } } , }
\end{array} \quad \text { and } \quad \left\{\begin{array}{rlrl}
\Delta v_{2}(\theta) & =0 & & \text { in } \Omega, \\
\partial_{\nu} v_{2}(\theta) & =0 & & \text { on } \Gamma, \\
v_{2}(\theta) & =w(\theta) & & \text { on } \Gamma_{\mathrm{c}} .
\end{array}\right.\right.
$$

We will prove the following result (see Section 4).

\footnotetext{
${ }^{2}$ For the well-posedness of all the Laplace's problems considered in the study, we refer to Appendix A
} 
Theorem 1.4. The problem of minimizing $\mathscr{F}$ over $\mathrm{H}_{\diamond}^{-1 / 2}(\partial \Omega)$ is a well-posed problem: there exists a unique $\theta_{\mathrm{o}} \in \mathrm{H}_{\diamond}^{-1 / 2}(\partial \Omega)$ such that

$$
\mathscr{F}\left(\theta_{\mathrm{O}}\right)=\min _{\theta \in \mathrm{H}_{\diamond}^{-1 / 2}(\partial \Omega)} \mathscr{F}(\theta) .
$$

Obviously, this optimal $\theta_{\mathrm{o}}$ depends on $\delta$, but in the following we forget the dependency in order to simplify notations. We define

$$
\varphi_{\mathrm{o}}=\partial_{\nu} w\left(\theta_{\mathrm{o}}\right)_{\mid \Gamma_{\mathrm{c}}} \quad \text { and } \quad \psi_{\mathrm{o}}=w\left(\theta_{\mathrm{o}}\right)_{\mid \Gamma_{\mathrm{c}}},
$$

where $w\left(\theta_{\mathrm{o}}\right)$ is defined by (1.4), and $u_{\mathrm{o}} \in \mathrm{H}^{1}(\Omega)$ verifies

$$
\left\{\begin{aligned}
\Delta u_{\mathrm{o}} & =0 & & \text { in } \Omega \\
u_{\mathrm{o}} & =g_{\mathrm{D}}^{\delta} & & \text { on } \Gamma \\
\partial_{\nu} u_{\mathrm{o}} & =\varphi_{\mathrm{o}} & & \text { on } \Gamma_{\mathrm{c}} .
\end{aligned}\right.
$$

Notice that $\varphi_{\mathrm{o}}, \psi_{\mathrm{o}}$ and $u_{\mathrm{o}}$ depend again on $\delta$, but we also forget this dependency for simplicity. We will prove the following two results (see Section 4).

Theorem 1.5. For all $\delta>0$ and $\boldsymbol{F}^{\delta} \in \mathbf{L}^{2}(\Omega)$ satisfying 1.3 , we have

$$
\left\|\nabla v_{1}\left(\theta_{\mathrm{O}}\right)-\nabla v_{2}\left(\theta_{\mathrm{O}}\right)-\boldsymbol{F}^{\delta}\right\|_{\mathbf{L}^{2}(\Omega)}=c \delta .
$$

Theorem 1.6. The triplet $\left(\varphi_{\mathrm{o}}, \psi_{\mathrm{o}}, u_{\mathrm{o}}\right)$ converges to $\left(\varphi_{\mathrm{ex}}, \psi_{\mathrm{ex}}, u_{\mathrm{ex}}\right)$ as $\delta$ converges to zero, strongly in $\mathrm{H}^{-1 / 2}\left(\Gamma_{\mathrm{c}}\right) \times \tilde{\mathrm{H}}^{1 / 2}\left(\Gamma_{\mathrm{c}}\right) \times \mathrm{H}^{1}(\Omega)$, where $\tilde{\mathrm{H}}^{1 / 2}\left(\Gamma_{\mathrm{c}}\right)$ is the quotient space $\mathrm{H}^{1 / 2}\left(\Gamma_{\mathrm{c}}\right) / \mathbb{R}$.

Because of these two results, we consider the triplet $\left(\varphi_{\mathrm{o}}, \psi_{\mathrm{o}}, u_{\mathrm{o}}\right)$ as our regularized solution to Problem (1.1), $u_{\mathrm{o}}$ being an approximation of the exact solution $u_{\mathrm{ex}}$ in $\Omega$. Actually, Theorem 1.5 implies that the couple $\left(\varphi_{\mathrm{o}}, \psi_{\mathrm{o}}\right)$ satisfies the well-known Morozov discrepancy principle, while Theorem 1.6 ensure the convergence of the approximated solution to the exact one as the amplitude of noise goes to zero.

Hence, to obtain our regularized solution, we only need to minimize the functional $\mathscr{F}$ over the space $\mathrm{H}_{\diamond}^{-1 / 2}(\partial \Omega)$, which is an unconstrained minimization problem easy to solve numerically. Note also that this is a method without regularization parameter, which automatically construct a solution satisfying the Morozov discrepancy principle with respect to the noisy data. These are the two main advantages and novelties of our method.

Link with the Kohn-Vogelius strategy. We now link the minimization problem of Theorem 1.4 with the well-known Kohn-Vogelius strategy, which is a regularization method for Problem (1.1) based on the minimization of a Kohn-Vogelius functional. Introduced in [5] to stabilize Problem (1.1), it has since been widely used in the context of inverse problems (see, among others, [1, 2, 4, 7, 19, 22, 38, and the references therein).

There are several variations of the Kohn-Vogelius strategy to handle Problem (1.1), depending on the choices of limit conditions in the auxiliary volumic problems. In the present paper, we focus on the one used in 21] to deal with inverse obstacle problem for Laplace's equation. More precisely, for $\varphi \in \mathrm{H}^{-1 / 2}\left(\Gamma_{\mathrm{c}}\right)$ and $\psi \in \mathrm{H}_{\diamond}^{1 / 2}\left(\Gamma_{\mathrm{c}}\right)$, where

$$
\mathrm{H}_{\diamond}^{1 / 2}\left(\Gamma_{\mathrm{c}}\right)=\left\{g \in \mathrm{H}^{1 / 2}\left(\Gamma_{\mathrm{c}}\right), \int_{\Gamma_{\mathrm{c}}} g \mathrm{~d} s=0\right\},
$$

we denote $v_{\varphi}$ and $v_{\psi}$ the two elements of $\mathrm{H}^{1}(\Omega)$ verifying respectively

$$
\left\{\begin{array} { r l l } 
{ \Delta v _ { \varphi } = 0 } & { \text { in } \Omega , } \\
{ v _ { \varphi } = 0 } & { \text { on } \Gamma , } \\
{ \partial _ { \nu } v _ { \varphi } = \varphi } & { \text { on } \Gamma _ { \mathrm { c } } , }
\end{array} \quad \text { and } \quad \left\{\begin{array}{rlrl}
\Delta v_{\psi} & =0 & & \text { in } \Omega, \\
\partial_{\nu} v_{\psi} & =0 & & \text { on } \Gamma, \\
v_{\psi} & =\psi & & \text { on } \Gamma_{\mathrm{c}} .
\end{array}\right.\right.
$$


Then the regularized Kohn-Vogelius functional writes, for $\varepsilon>0$ and for all $(\varphi, \psi) \in \mathrm{H}^{-1 / 2}\left(\Gamma_{\mathrm{c}}\right) \times \mathrm{H}_{\diamond}^{1 / 2}\left(\Gamma_{\mathrm{c}}\right)$, as

$$
\mathscr{K} \mathscr{V}(\varphi, \psi)=\frac{1}{2} \int_{\Omega}\left|\nabla v_{\varphi}-\nabla v_{\psi}-\boldsymbol{F}^{\delta}\right|^{2} \mathrm{~d} x+\frac{\varepsilon}{2} \int_{\Omega}\left(\left|\nabla v_{\varphi}\right|^{2}+\left|\nabla v_{\psi}\right|^{2}\right) \mathrm{d} x
$$

In this form, it clearly appears to be a Tikhonov functional, and indeed it always has a unique minimizer (see [21, Proposition 2.5]):

Proposition 1.7. For all $\varepsilon>0$, the functional $\mathscr{K} \mathscr{V}$ admits a unique minimizer $\left(\varphi_{\varepsilon}, \psi_{\varepsilon}\right)$ over the space $\mathrm{H}^{-1 / 2}\left(\Gamma_{\mathrm{c}}\right) \times \mathrm{H}_{\diamond}^{1 / 2}\left(\Gamma_{\mathrm{c}}\right)$.

Remark 1.8. Notice that the above Kohn-Vogelius functional can be written equivalently in the more classical form

$$
\mathscr{K} \mathscr{V}(\varphi, \psi)=\frac{1}{2} \int_{\Omega}\left|\nabla\left(v_{\varphi}+u_{\mathrm{D}}^{\delta}\right)-\nabla\left(v_{\psi}+u_{\mathrm{N}}^{\delta}\right)\right|^{2} \mathrm{~d} x+\frac{\varepsilon}{2} \int_{\Omega}\left(\left|\nabla v_{\varphi}\right|^{2}+\left|\nabla v_{\psi}\right|^{2}\right) \mathrm{d} x .
$$

As usual in inverse problems, one of the main question is then to set the parameter of regularization with respect to the a priori known amplitude of noise. We have the following result, basically saying that the Morozov discrepancy principle is a viable method to do so (see [21, Proposition 2.8]):

Theorem 1.9. There exists a unique $\varepsilon=\varepsilon(\delta)>0$ so that the corresponding minimizer $\left(\varphi_{\varepsilon(\delta)}, \psi_{\varepsilon(\delta)}\right)$ of $\mathscr{K} \mathscr{V}$, which belongs to $\mathrm{H}^{-1 / 2}\left(\Gamma_{\mathrm{c}}\right) \times \mathrm{H}_{\diamond}^{1 / 2}\left(\Gamma_{\mathrm{c}}\right)$, satisfies the Morozov discrepancy principle

$$
\left\|\nabla v_{\varphi_{\varepsilon(\delta)}}-\nabla v_{\psi_{\varepsilon(\delta)}}-\boldsymbol{F}^{\delta}\right\|_{\mathbf{L}^{2}(\Omega)}=c \delta .
$$

Furthermore, $\left(\varphi_{\varepsilon(\delta)}, \psi_{\varepsilon(\delta)}\right)$ converges to $\left(\varphi_{\mathrm{ex}}, \psi_{\mathrm{ex}}\right)$ strongly in $\mathrm{H}^{-1 / 2}\left(\Gamma_{\mathrm{c}}\right) \times \tilde{\mathrm{H}}^{1 / 2}\left(\Gamma_{\mathrm{c}}\right)$ when $\delta$ goes to zero.

It turns out that $\left(\varphi_{\mathrm{o}}, \psi_{\mathrm{o}}\right)$ is precisely the minimizer of $\mathscr{K} \mathscr{V}$ corresponding to $\varepsilon(\delta)$ (see the proof of the following result in Section 4p:

Theorem 1.10. We have

$$
\varepsilon(\delta)=\frac{c \delta}{\sqrt{\int_{\Omega}\left(\left|\nabla v_{\varphi_{\mathrm{o}}}\right|^{2}+\left|\nabla v_{\psi_{\mathrm{o}}}\right|^{2}\right) \mathrm{d} x}} \quad \text { and } \quad\left(\varphi_{\mathrm{o}}, \psi_{\mathrm{o}}\right)=\left(\varphi_{\varepsilon(\delta)}, \psi_{\varepsilon(\delta)}\right)
$$

Hence, minimizing the functional $\mathscr{F}$ is not only a method to stably obtain a good reconstruction of the missing data in Problem (1.1), but also a method to find the minimizer of $\mathscr{K} \mathscr{V}$ and to determine the value of the parameter of regularization in the Kohn-Vogelius strategy satisfying the Morozov discrepancy principle. This represents the last main result of our work.

Outline. The paper is organized as follows. In Section 2, we study an operator used in the following sections. In Section 3, we prove all the main results in an abstract setting, that we apply in Section 4 to our problem of interest, proving in particular Theorem 1.4 . Theorem 1.5 , Theorem 1.6 and Theorem 1.10. Section 5 is dedicated to numerical exemples in two-dimensional and three-dimensional settings, showing the feasibility and efficiency of the proposed method. In Section 6 we present some final comments, in particular on the rate of convergence of the method, and on how to impose exactly a finite number of constraints on the solution. Finally, in Appendix $\mathrm{A}$, we precise the different functional settings used in the study.

Aknowledgements. We are grateful to Sylvain Ervedoza for sharing previous versions of his work 31, which inspires us the Section 6.2 We also express our gratitude to Sophie Jan, for enriching conversations on optimization in infinite dimensional spaces. 


\section{On an operator from the boundary to the volume}

The operator

$$
A:(\varphi, \psi) \in \mathrm{H}^{-1 / 2}\left(\Gamma_{\mathrm{c}}\right) \times \mathrm{H}_{\diamond}^{1 / 2}\left(\Gamma_{\mathrm{c}}\right) \longmapsto \nabla v_{\varphi}-\nabla v_{\psi} \in \mathbf{H}(\Omega),
$$

where $v_{\varphi}$ and $v_{\psi}$ are defined by (1.7), and where

$$
\mathbf{H}(\Omega)=\left\{\nabla w, w \in \mathrm{H}^{1}(\Omega) \text { satisfies } \Delta w=0 \text { in } \Omega\right\},
$$

plays a central role in our study. From Lemmata A.2 and A.3, we know that the bilinear application on $\mathrm{H}^{-1 / 2}\left(\Gamma_{\mathrm{c}}\right) \times \mathrm{H}_{\diamond}^{1 / 2}\left(\Gamma_{\mathrm{c}}\right)$

$$
\left\{\left(\varphi_{1}, \psi_{1}\right),\left(\varphi_{2}, \psi_{2}\right)\right\} \longmapsto \int_{\Omega}\left(\nabla v_{\varphi_{1}} \cdot \nabla v_{\varphi_{2}}+\nabla v_{\psi_{1}} \cdot \nabla v_{\psi_{2}}\right) \mathrm{d} x
$$

is a scalar product, the corresponding norm being equivalent to the standard norm on the space $\mathrm{H}^{-1 / 2}\left(\Gamma_{\mathrm{c}}\right) \times \mathrm{H}_{\diamond}^{1 / 2}\left(\Gamma_{\mathrm{c}}\right)$, so that $\mathrm{H}^{-1 / 2}\left(\Gamma_{\mathrm{c}}\right) \times \mathrm{H}_{\diamond}^{1 / 2}\left(\Gamma_{\mathrm{c}}\right)$ endowed with this scalar product is a Hilbert space. Similarly, from Lemma A.4, $\mathbf{H}(\Omega)$ is a Hilbert space when endowed with the standard $\mathbf{L}^{2}-$ scalar product.

We first have the following properties.

Proposition 2.1. $\operatorname{Ker}(A)=\{(0,0)\}$, Range $(A) \neq \mathbf{H}(\Omega)$ and $\overline{\operatorname{Range}(\mathrm{A})}=\mathbf{H}(\Omega)$.

Proof. Firstly let $(\varphi, \psi) \in \mathrm{H}^{-1 / 2}\left(\Gamma_{\mathrm{c}}\right) \times \mathrm{H}_{\diamond}^{1 / 2}\left(\Gamma_{\mathrm{c}}\right)$ be such that $A(\varphi, \psi)=0$, that is $\nabla v_{\varphi}-\nabla v_{\psi}=0$. There exists $\alpha \in \mathbb{R}$ such that $v_{\varphi}=v_{\psi}+\alpha$. Then

$$
\int_{\Gamma_{\mathrm{c}}} \psi \mathrm{d} s=\int_{\Gamma_{\mathrm{c}}} v_{\psi} \mathrm{d} s=0 \Rightarrow \alpha=\frac{1}{\left|\Gamma_{\mathrm{c}}\right|} \int_{\Gamma_{\mathrm{c}}} v_{\varphi} \mathrm{d} s
$$

It is clear that

$$
\partial_{\nu} v_{\varphi \mid \Gamma}=\partial_{\nu}\left(v_{\psi}+\alpha\right)_{\mid \Gamma}=\partial_{\nu} v_{\psi \mid \Gamma}=0
$$

As also $\Delta v_{\varphi}=0$ and $v_{\varphi \mid \Gamma}=0$, we have $v_{\varphi}=0$. Hence $\varphi=0$ and $\alpha=0$. As a consequence, we have $v_{\psi}=v_{\varphi}-\alpha=0$, so $\psi=0$.

Secondly, for $\left(g_{\mathrm{D}}, g_{\mathrm{N}}\right) \in \mathrm{H}^{1 / 2}(\Gamma) \times \mathrm{H}^{-1 / 2}(\Gamma)$ such that problem 1.1 fails to have a solution, we define $\boldsymbol{F}=\nabla u_{\mathrm{N}}-\nabla u_{\mathrm{D}} \in \mathbf{H}(\Omega)$. If there would exist $(\varphi, \psi) \in \mathrm{H}^{-1 / 2}\left(\Gamma_{\mathrm{c}}\right) \times \mathrm{H}_{\diamond}^{1 / 2}\left(\Gamma_{\mathrm{c}}\right)$ such that we have $A(\varphi, \psi)=\boldsymbol{F}$, we would get $\nabla\left(v_{\varphi}+u_{\mathrm{D}}\right)=\nabla\left(v_{\psi}+u_{\mathrm{N}}\right)$ in $\Omega$. Therefore, there would exist $\alpha \in \mathbb{R}$ such that $v_{\varphi}+u_{\mathrm{D}}=v_{\psi}+u_{\mathrm{N}}+\alpha$, leading to

$$
\left\{\begin{aligned}
\Delta\left(v_{\varphi}+u_{\mathrm{D}}\right) & = & & \text { in } \Omega \\
v_{\varphi}+u_{\mathrm{D}} & =g_{\mathrm{D}} & & \text { on } \Gamma \\
\partial_{\nu}\left(v_{\varphi}+u_{\mathrm{D}}\right) & =g_{\mathrm{N}} & & \text { on } \Gamma_{\mathrm{c}} .
\end{aligned}\right.
$$

In other words, $v_{\varphi}+u_{\mathrm{D}}$ verifies 1.1 , leading to a contradiction. Hence $\operatorname{Range}(A) \neq \mathbf{H}(\Omega)$.

Finally, let $\boldsymbol{p} \in \mathbf{H}(\Omega)$ be such that for all $(\varphi, \psi) \in \mathrm{H}^{-1 / 2}\left(\Gamma_{\mathrm{c}}\right) \times \mathrm{H}_{\diamond}^{1 / 2}\left(\Gamma_{\mathrm{c}}\right)$, we have

$$
(A(\varphi, \psi), \boldsymbol{p})_{\mathbf{L}^{2}(\Omega)}=0 \Longleftrightarrow \int_{\Omega} \nabla\left(v_{\varphi}-v_{\psi}\right) \cdot \boldsymbol{p} \mathrm{d} x=0 .
$$

Let us prove that $\boldsymbol{p}=\mathbf{0}$ which implies that Range $(A)^{\perp}=\{\mathbf{0}\}$ and then, using the classical density criteria (i.e. a corollary of the Hahn-Banach theorem in Hilbert spaces), we will obtain $\overline{\operatorname{Range}(A)}=\mathbf{H}(\Omega)$. There exists $w \in \mathrm{H}^{1}(\Omega)$, harmonic in $\Omega$, such that $\boldsymbol{p}=\nabla w$. So $w$ verifies

$$
\int_{\Omega} \nabla\left(v_{\varphi}-v_{\psi}\right) \cdot \nabla w \mathrm{~d} x=0, \quad \forall(\varphi, \psi) \in \mathrm{H}^{-1 / 2}\left(\Gamma_{\mathrm{c}}\right) \times \mathrm{H}_{\diamond}^{1 / 2}\left(\Gamma_{\mathrm{c}}\right) .
$$


For $\theta \in C_{c}^{\infty}\left(\Gamma_{\mathrm{c}}\right)$, we define $h \in \mathrm{H}^{1}(\Omega)$ as the unique solution of

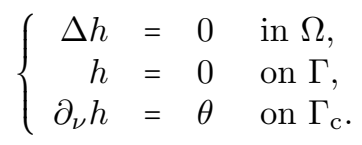

Setting $\varphi=\partial_{\nu} h_{\mid \Gamma_{\mathrm{c}}}$, it is readily seen that $v_{\varphi}=h$. So choosing also $\psi=0$ so that $v_{\psi}=0$, we obtain

$$
0=\int_{\Omega} \nabla\left(v_{\varphi}-v_{\psi}\right) \cdot \nabla w \mathrm{~d} x=\int_{\Omega} \nabla v_{\varphi} \cdot \nabla w \mathrm{~d} x=\int_{\Omega} \nabla h \cdot \nabla w \mathrm{~d} x=\left\langle\partial_{\nu} w, \theta\right\rangle_{\Gamma_{\mathrm{c}}} .
$$

Since this equality holds for all $\theta \in C_{c}^{\infty}\left(\Gamma_{\mathrm{c}}\right)$, it follows $\partial_{\nu} w_{\mid \Gamma_{\mathrm{c}}}=0$. Now, for $\theta \in C_{c}^{\infty}\left(\Gamma_{\mathrm{c}}\right)$, we define $h \in \mathrm{H}^{1}(\Omega)$ the solution of

$$
\left\{\begin{aligned}
\Delta h & =0 & & \text { in } \Omega, \\
\partial_{\nu} h & =0 & & \text { on } \Gamma, \\
h & =\theta-\frac{1}{\left|\Gamma_{\mathrm{c}}\right|} \int_{\Gamma_{\mathrm{c}}} \theta \mathrm{d} s & & \text { on } \Gamma_{\mathrm{c}} .
\end{aligned}\right.
$$

Note that such a function $h$ is determined only up to a constant, which is without consequences for what follows. We define

$$
\psi=h_{\mid \Gamma_{\mathrm{c}}}-\frac{1}{\left|\Gamma_{\mathrm{c}}\right|} \int_{\Gamma_{\mathrm{c}}} h \mathrm{~d} s
$$

which belongs to $\mathrm{H}_{\diamond}^{1 / 2}\left(\Gamma_{\mathrm{c}}\right)$. Then $\nabla v_{\psi}=\nabla h$, so choosing $\varphi=0$ so that $v_{\varphi}=0$, we obtain

$$
0=\int_{\Omega} \nabla v_{\psi} \cdot \nabla w \mathrm{~d} x=\int_{\Omega} \nabla h \cdot \nabla w \mathrm{~d} x=\int_{\Gamma_{\mathrm{c}}} \theta\left(w-\frac{1}{\left|\Gamma_{\mathrm{c}}\right|} \int_{\Gamma_{\mathrm{c}}} w \mathrm{~d} s\right) \mathrm{d} x=\int_{\Gamma_{\mathrm{c}}} w\left(\theta-\frac{1}{\left|\Gamma_{\mathrm{c}}\right|} \int_{\Gamma_{\mathrm{c}}} \theta \mathrm{d} s\right) \mathrm{d} x .
$$

Since this equality holds for all $\theta \in C_{c}^{\infty}\left(\Gamma_{\mathrm{c}}\right)$, it follows $w_{\mid \Gamma_{\mathrm{c}}}=\frac{1}{\left|\Gamma_{\mathrm{c}}\right|} \int_{\Gamma_{\mathrm{c}}} w \mathrm{~d} s$. As a conclusion, as $w$ verifies $\Delta w=0, \partial_{\nu} w_{\mid \Gamma_{\mathrm{c}}}=0$ and $w_{\mid \Gamma_{\mathrm{c}}}=\alpha \in \mathbb{R}$, we obtain $w=\alpha$ in $\Omega$. Hence $\boldsymbol{p}=\nabla w=\mathbf{0}$, which ends the proof.

We can now focus on $A^{*}$, the adjoint of $A$, which as usually is defined by the relation

$$
(A(\varphi, \psi), \boldsymbol{p})_{\mathbf{L}^{2}(\Omega)}=\left\{(\varphi, \psi), A^{*} \boldsymbol{p}\right\}, \quad \forall(\varphi, \psi) \in \mathrm{H}^{-1 / 2}\left(\Gamma_{\mathrm{c}}\right) \times \mathrm{H}_{\diamond}^{1 / 2}\left(\Gamma_{\mathrm{c}}\right), \forall \boldsymbol{p} \in \mathbf{H}(\Omega) .
$$

Proposition 2.2. Let $\boldsymbol{p} \in \mathbf{H}(\Omega)$, so that there exists $w \in \mathrm{H}^{1}(\Omega)$ such that $p=\nabla w$ with $\Delta w=0$ in $\Omega$. Then we have

$$
A^{*} p=\left(\varphi_{p}, \psi_{p}\right), \quad \text { with } \quad \varphi_{p}=\partial_{\nu} w_{\mid \Gamma_{\mathrm{c}}} \quad \text { and } \quad \psi_{p}=-\left(w_{\mid \Gamma_{\mathrm{c}}}-\frac{1}{\left|\Gamma_{\mathrm{c}}\right|} \int_{\Gamma_{\mathrm{c}}} w \mathrm{~d} s\right) .
$$

Proof. Let $\boldsymbol{p} \in \mathbf{H}(\Omega)$, and $A^{*} \boldsymbol{p}=\left(\varphi_{p}, \psi_{p}\right)$ in $\mathrm{H}^{-1 / 2}\left(\Gamma_{\mathrm{c}}\right) \times \mathrm{H}_{\diamond}^{1 / 2}\left(\Gamma_{\mathrm{c}}\right)$. There exists $w \in \mathrm{H}^{1}(\Omega)$ verifying $\Delta w=0$ and $\nabla w=\boldsymbol{p}$. For any $\varphi \in \mathrm{H}^{-1 / 2}\left(\Gamma_{\mathrm{c}}\right)$, we have

$$
\int_{\Omega} \nabla v_{\varphi} \cdot \nabla w \mathrm{~d} x=(A(\varphi, 0), \boldsymbol{p})_{\mathbf{L}^{2}(\Omega)}=\left\{(\varphi, 0), A^{*} \boldsymbol{p}\right\}=\int_{\Omega} \nabla v_{\varphi} \cdot \nabla v_{\varphi_{p}} \mathrm{~d} x .
$$

For $\theta \in C_{c}^{\infty}\left(\Gamma_{\mathrm{c}}\right)$, let $h \in \mathrm{H}^{1}(\Omega)$ be the unique solution of

$$
\left\{\begin{array}{rll}
\Delta h=0 & & \text { in } \Omega, \\
h=0 & & \text { on } \Gamma, \\
h=\theta & & \text { on } \Gamma_{\mathrm{c}} .
\end{array}\right.
$$


Defining $\varphi=\partial_{\nu} h_{\mid \Gamma_{\mathrm{c}}}$, it is readily seen that $v_{\varphi}=h$. This easily leads to

$$
\left\langle\partial_{\nu} w, \theta\right\rangle_{\Gamma_{\mathrm{c}}}=\int_{\Omega} \nabla v_{\varphi} \cdot \nabla w \mathrm{~d} x=\int_{\Omega} \nabla v_{\varphi} \cdot \nabla v_{\varphi_{p}} \mathrm{~d} x=\left\langle\varphi_{p}, \theta\right\rangle_{\Gamma_{\mathrm{c}}} .
$$

Since this equality holds for all $\theta \in C_{c}^{\infty}\left(\Gamma_{\mathrm{c}}\right)$, it follows $\varphi_{p}=\partial_{\nu} w_{\mid \Gamma_{\mathrm{c}}}$.

Now, for any $\psi \in \mathrm{H}_{\diamond}^{1 / 2}\left(\Gamma_{\mathrm{c}}\right)$, we have

$$
\int_{\Omega} \nabla v_{\psi} \cdot \nabla w \mathrm{~d} x=-(A(0, \psi), \boldsymbol{p})_{\mathbf{L}^{2}(\Omega)}=-\left\{(0, \psi), A^{*} \boldsymbol{p}\right\}=-\int_{\Omega} \nabla v_{\psi} \cdot \nabla v_{\psi_{p}} \mathrm{~d} x .
$$

For $\theta \in C_{c}^{\infty}\left(\Gamma_{\mathrm{c}}\right)$, let $h \in \mathrm{H}^{1}(\Omega)$ be a solution of

$$
\left\{\begin{aligned}
\Delta h & =0 & & \text { in } \Omega, \\
\partial_{\nu} h & =0 & & \text { on } \Gamma, \\
\partial_{\nu} h & =\theta-\frac{1}{\left|\Gamma_{\mathrm{c}}\right|} \int_{\Gamma_{\mathrm{c}}} \theta \mathrm{d} s & & \text { on } \Gamma_{\mathrm{c}} .
\end{aligned}\right.
$$

Setting

$$
\psi=h_{\mid \Gamma_{\mathrm{c}}}-\frac{1}{\left|\Gamma_{\mathrm{c}}\right|} \int_{\Gamma_{\mathrm{c}}} h \mathrm{~d} s \in \mathrm{H}_{\diamond}^{1 / 2}\left(\Gamma_{\mathrm{c}}\right),
$$

we clearly have $\nabla v_{\psi}=\nabla h$ and then $v_{\psi}=h+\alpha$ with $\alpha \in \mathbb{R}$, so that

$$
\begin{aligned}
\int_{\Gamma_{\mathrm{c}}} \theta\left(w-\frac{1}{\left|\Gamma_{\mathrm{c}}\right|} \int_{\Gamma_{\mathrm{c}}} w \mathrm{~d} s\right) \mathrm{d} s=\int_{\Gamma_{\mathrm{c}}} w\left(\theta-\frac{1}{\left|\Gamma_{\mathrm{c}}\right|} \int_{\Gamma_{\mathrm{c}}} \theta \mathrm{d} s\right) \mathrm{d} s= \\
\quad\left\langle\partial_{\nu} h, w\right\rangle=\int_{\Omega} \nabla v_{\psi} \cdot \nabla w \mathrm{~d} x=-\int_{\Omega} \nabla v_{\psi} \cdot \nabla v_{\psi_{p}} \mathrm{~d} x=-\int_{\Gamma_{\mathrm{c}}} \theta \psi_{p} \mathrm{~d} x,
\end{aligned}
$$

the last equality coming from the fact that $\psi_{p}$ is by definition mean-free on $\Gamma_{\mathrm{c}}$. Hence

$$
\psi_{p}=-\left(w-\frac{1}{\left|\Gamma_{\mathrm{c}}\right|} \int_{\Gamma_{\mathrm{c}}} w \mathrm{~d} s\right),
$$

which ends the proof.

Remark 2.3. Note that $A^{*}$ is a one-to-one operator, as expected as $\overline{\operatorname{Range}(\mathrm{A})}=\mathbf{H}(\Omega)$. Indeed, if $A^{*} \boldsymbol{p}=(0,0)$, then any $w \in \mathrm{H}^{1}(\Omega)$ verifying $\nabla w=\boldsymbol{p}$ and $\Delta w=0$ is a constant function in $\Omega$, and therefore $\boldsymbol{p}=\mathbf{0}$.

\section{Abstract setting}

We now present the main results of our work in an abstract setting, that we will later apply to our problem of interest. The strategy described below is a generalization of the one developed in [14 for the quasi-reversibility, with a point of view which is in a sense reversed, as our primal problem here is the dual problem in [14. This is also closely related to the works on control theory [28, 31.

Let $\mathscr{X}, \mathscr{Y}$ be two Hilbert spaces with scalar products $(\cdot, \cdot) \mathscr{X}$ and $(\cdot, \cdot) \mathscr{Y}$ and corresponding norms $\|\cdot\|_{\mathscr{X}}$ and $\|\cdot\|_{\mathscr{Y}}$. Let $\mathscr{A}$ be a linear continuous operator from $\mathscr{X}$ to $\mathscr{Y}$, such that $\operatorname{Ker}(\mathscr{A})=0 \mathscr{Y}$, $\operatorname{Range}(\mathscr{A}) \neq \mathscr{Y}$ but $\overline{\operatorname{Range}(\mathscr{A})}=\mathscr{Y}$. Then $\mathscr{A}^{*}$ is well defined as a linear continuous operator from $\mathscr{Y}$ to $\mathscr{X}$, and is one-to-one.

Remark 3.1. Obviously, in next section, we will choose $\mathscr{X}=\mathrm{H}^{-1 / 2}\left(\Gamma_{\mathrm{c}}\right) \times \mathrm{H}_{\diamond}^{1 / 2}\left(\Gamma_{\mathrm{c}}\right), \mathscr{Y}=\mathbf{H}(\Omega)$ and $\mathscr{A}=A$. 
For $y \in \mathscr{Y}$, the problem of finding some $x \in \mathscr{X}$ such that $\mathscr{A} x=y$ is ill-posed, as by definition it may fail to have a solution. Let $y_{\mathrm{s}}$ be in the range of $\mathscr{A}, x_{\mathrm{s}}$ be the only element of $\mathscr{X}$ such that

$$
\mathscr{A} x_{\mathrm{s}}=y_{\mathrm{s}},
$$

and $y^{\eta}$ in $\mathscr{Y}$ be such that

$$
\left\|y^{\eta}-y_{\mathrm{s}}\right\|_{\mathscr{Y}} \leqslant \eta,
$$

for some $\eta>0$. Here $y_{\mathrm{s}}$ has to be understood as an exact data, $x_{\mathrm{s}}$ the corresponding exact solution, $y^{\eta}$ a noisy data for our problem, and $\eta$ is the supposedly known amplitude of noise on the data.

As $\mathscr{A}$ is not onto, they may have no $x$ in $\mathscr{X}$ such that $\mathscr{A} x=y^{\eta}$. Thus it is not judicious to use a usual least-squares approach which consists in minimizing $\frac{1}{2}\left\|A x-y^{\eta}\right\|_{\mathscr{Y}}^{2}$, even if this is the main problem on which we want to focus on. However, the set

$$
\mathscr{M}=\left\{x \in \mathscr{X},\left\|\mathscr{A} x-y^{\eta}\right\|_{\mathscr{Y}} \leqslant \eta\right\},
$$

i.e. the set of element of $\mathscr{X}$ satisfying the Morozov discrepancy principle, is not empty, as $x_{\mathrm{s}}$ belongs to $\mathscr{M}$. We now aim to construct from $y^{\eta}$ one element of this set, stably, without other parameters than $\eta$ and the noisy data itself, and in such a way that the lower the amplitude of noise is, the closer it is to the exact solution $x_{\mathrm{s}}$.

To do so, we start by solving a well-posed minimization problem not in the space $\mathscr{X}$ of the solutions, but in the space $\mathscr{Y}$ of the data. It is in that sense that the regularization method is a dual strategy.

\subsection{A minimization problem}

We define a functional acting on $\mathscr{Y}$ :

$$
\mathscr{J}: y \in \mathscr{Y} \longmapsto \frac{1}{2}\left\|\mathscr{A}^{*} y\right\|_{\mathscr{X}}^{2}+\eta\|y\|_{\mathscr{Y}}-\left(y, y^{\eta}\right)_{\mathscr{Y}}
$$

This functional is clearly continuous, and it is also strictly convex as $\mathscr{A}^{*}$ is one-to-one.

Proposition 3.2. The functional $\mathscr{J}$ is coercive, i.e.

$$
\lim _{\|y\|_{\mathscr{Y} \rightarrow \infty}} \mathscr{J}(y)=\infty .
$$

Proof. Suppose it is not the case. Then it exists a sequence $\left(y_{n}\right)_{n \in \mathbb{N}}$ of elements of $\mathscr{Y}$ and a constant $C \in \mathbb{R}$ such that

$$
\lim _{n \rightarrow \infty}\left\|y_{n}\right\|_{\mathscr{Y}}=\infty \quad \text { and } \quad \mathscr{J}\left(y_{n}\right)<C .
$$

Define, for all $n \in \mathbb{N}, z_{n}=y_{n}\left\|y_{n}\right\|_{\mathscr{O}}^{-1}$, which is obviously a bounded sequence. Therefore, one can extract from $\left(z_{n}\right)_{n \in \mathbb{N}}$ a subsequence weakly converging to some $z$ in $\mathscr{Y}$. We still denote $\left(z_{n}\right)_{n \in \mathbb{N}}$ this subsequence. As $\mathscr{A}^{*}$ is a linear operator, $\mathscr{A}^{*} z_{n}$ converges to $\mathscr{A}^{*} z$. From this and since

$$
\frac{1}{2}\left\|\mathscr{A}^{*} z_{n}\right\|_{\mathscr{X}}^{2}+\frac{1}{\left\|y_{n}\right\|_{\mathscr{Y}}}\left[\eta-\left(z_{n}, y^{\eta}\right) \mathscr{Y}\right]<\frac{C}{\left\|y_{n}\right\|_{\mathscr{Y}}^{2}},
$$

we obtain that $\mathscr{A}^{*} z=0 \mathscr{X}$, leading immediately to $z=0_{\mathscr{Y}}$. Note that in particular we have

$$
\lim _{n \rightarrow \infty}\left(z_{n}, y^{\eta}\right)_{\mathscr{Y}}=0 .
$$

As in addition

$$
\mathscr{J}\left(y_{n}\right)>\left\|y_{n}\right\|_{\mathscr{Y}}\left[\eta-\left(z_{n}, y^{\eta}\right) \mathscr{Y}\right],
$$

we obtain a contradiction by letting $n$ goes to infinity. 
As $\mathscr{J}$ is continuous, strictly convex and coercive, we know (see, e.g., [29, Proposition 1.2 p.35]) that there existe a unique $y_{\mathrm{o}} \in \mathscr{Y}$ such that

$$
y_{\mathrm{o}}=\underset{y \in \mathscr{Y}}{\arg \min } \mathscr{J}(y) .
$$

Lemma 3.3. $y_{\mathrm{o}}=0_{\mathscr{Y}}$ if and only if $\left\|y^{\eta}\right\|_{\mathscr{Y}} \leqslant \eta$.

Proof. For any $\beta>0$, one has

$$
\mathscr{J}\left(\beta y^{\eta}\right)=\frac{\beta^{2}}{2}\left\|\mathscr{A}^{*} y^{\eta}\right\|_{\mathscr{X}}^{2}+\beta\left\|y^{\eta}\right\| \mathscr{Y}\left[\eta-\left\|y^{\eta}\right\| \mathscr{Y}\right] .
$$

Then, on the one hand, if $y_{\mathrm{o}}=0_{\mathscr{Y}}$, one has $\mathscr{J}\left(\beta y^{\eta}\right) \geqslant 0$ for all $\beta>0$, leading to $\left\|y^{\eta}\right\| \mathscr{Y}\left[\eta-\left\|y^{\eta}\right\|_{\mathscr{Y}}\right] \geqslant 0$ and finally $\eta \geqslant\left\|y^{\eta}\right\|_{\mathscr{Y}}$.

On the other hand, if $y_{\mathrm{o}} \neq 0_{\mathscr{Y}}$, then $\mathscr{J}\left(y_{\mathrm{o}}\right)<\mathscr{J}\left(0_{\mathscr{Y}}\right)=0$, implying in particular that

$$
\eta\left\|y_{\mathrm{o}}\right\| \mathscr{Y}<\left(y_{\mathrm{o}}, y^{\eta}\right)_{\mathscr{Y}}
$$

and hence $\left\|y^{\eta}\right\|_{\mathscr{Y}}>\eta$.

From now on we make the assumption that $\left\|y^{\eta}\right\|_{\mathscr{Y}}>\eta$, so that the minimum of $\mathscr{J}$ is not reached in $0 \mathscr{Y}$. Note that it is necessarily true for $\eta$ small enough, as by definition

$$
\left\|y^{\eta}-y_{\mathrm{s}}\right\|_{\mathscr{Y}} \leqslant \eta \Rightarrow\left\|y_{\mathrm{s}}\right\|_{\mathscr{Y}}-\eta \leqslant\left\|y^{\eta}\right\|_{\mathscr{Y}} .
$$

In other word, for all $\eta$ such that $2 \eta$ is strictly smaller than $\left\|y_{\mathrm{s}}\right\| \mathscr{Y}$, all below results apply, which is in particular the case when $\eta$ goes to zero.

Proposition 3.4. $y_{\mathrm{o}}$ is the minimizer of $\mathscr{J}$ if and only if

$$
\mathscr{A} \mathscr{A}^{*} y_{\mathrm{o}}+\eta \frac{y_{\mathrm{o}}}{\left\|y_{\mathrm{o}}\right\|_{\mathscr{Y}}}=y^{\eta} .
$$

Proof. This is just the Euler-Lagrange equation associated with $\mathscr{J}$, which is well-defined as soon as $y_{\mathrm{o}} \neq 0 \mathscr{Y}$.

\subsection{The regularized solution}

Definition and first properties. We are now in position to define our regularized solution to problem $\mathscr{A} x=y^{\eta}$. To do so, we define

$$
x_{\mathrm{o}}=\mathscr{A}^{*} y_{\mathrm{o}}
$$

which by definition is an element of $\mathscr{X}$. The previous Proposition 3.4 shows that

$$
\mathscr{A} x_{\mathrm{o}}=y^{\eta}-\eta \frac{y_{\mathrm{o}}}{\left\|y_{\mathrm{o}}\right\|_{\mathscr{Y}}}
$$

which implies in particular that

$$
\left\|\mathscr{A} x_{\mathrm{o}}-y^{\eta}\right\|_{\mathscr{Y}}=\eta .
$$

Hence, $x_{\mathrm{o}}$ belongs to $\mathscr{M}$ by construction. From now on, we consider $x_{\mathrm{o}}$ as our regularized solution. Note in particular that it is unique, exists regardless of the compatibility of the noisy data, and does not depend on any parameter except for the noise amplitude $\eta$ (and obviously the noisy data itself). Note also that it satisfies the regularized problem (3.4), so in some sense the right-hand side of (3.4) can be viewed as a regularized version of the data for which our main problem always have a (necessarily unique) solution.

Before looking at convergence properties as $\eta$ goes to zero, we prove some results about $x_{\mathrm{o}}$. 
Proposition 3.5. We have

$$
\left\|x_{\mathrm{o}}\right\|_{\mathscr{X}}^{2}=-2 \mathscr{J}\left(y_{\mathrm{o}}\right)
$$

Proof. One has

$$
\begin{aligned}
\mathscr{J}\left(y_{\mathrm{o}}\right) & =\frac{1}{2}\left\|\mathscr{A}^{*} y_{\mathrm{o}}\right\|_{\mathscr{X}}^{2}+\eta\left\|y_{\mathrm{o}}\right\| \mathscr{Y}-\left(y_{\mathrm{o}}, y^{\eta}\right)_{\mathscr{Y}} \\
& =\frac{1}{2}\left(x_{\mathrm{o}}, \mathscr{A}^{*} y_{\mathrm{o}}\right)_{\mathscr{X}}+\eta\left\|y_{\mathrm{o}}\right\| \mathscr{Y}-\left(y_{\mathrm{o}}, y^{\eta}\right)_{\mathscr{Y}} \\
& =\frac{1}{2}\left(\mathscr{A} x_{\mathrm{o}}, y_{\mathrm{o}}\right)_{\mathscr{Y}}+\eta\left\|y_{\mathrm{o}}\right\|_{\mathscr{Y}}-\left(y_{\mathrm{o}}, y^{\eta}\right)_{\mathscr{Y}} \\
& =\frac{1}{2}\left(y^{\eta}-\frac{\eta}{\left\|y_{\mathrm{o}}\right\| \mathscr{Y}} y_{\mathrm{o}}, y_{\mathrm{o}}\right)_{\mathscr{Y}}+\eta\left\|y_{\mathrm{o}}\right\| \mathscr{Y}-\left(y_{\mathrm{o}}, y^{\eta}\right)_{\mathscr{Y}} \\
& =\frac{\eta}{2}\left\|y_{\mathrm{o}}\right\| \mathscr{Y}-\frac{1}{2}\left(y_{\mathrm{o}}, y^{\eta}\right)_{\mathscr{Y}} .
\end{aligned}
$$

Therefore

$$
\mathscr{J}\left(y_{\mathrm{o}}\right)=\frac{1}{2}\left\|\mathscr{A}^{*} y_{\mathrm{o}}\right\|_{\mathscr{X}}^{2}+2 \mathscr{J}\left(y_{\mathrm{o}}\right)=\frac{1}{2}\left\|x_{\mathrm{o}}\right\|_{\mathscr{X}}^{2}+2 \mathscr{J}\left(y_{\mathrm{o}}\right),
$$

which ends the proof.

It turns out that by construction, among all $x \in \mathscr{M}, x_{\mathrm{o}}$ is the one of minimal norm (see the following proposition). In other word, $x_{\mathrm{o}}$ defined by (3.4), could be alternatively defined as

$$
x_{\mathrm{o}}=\underset{x \in \mathscr{M}}{\arg \min }\|x\|_{\mathscr{X}},
$$

which is precisely the point of view adopted in [14.

Proposition 3.6. Let $x \in \mathscr{M}, x \neq x_{\mathrm{o}}$. Then $\|x\|_{\mathscr{X}}>\left\|x_{\mathrm{o}}\right\|_{\mathscr{X}}$.

Proof. Let $x \in \mathscr{M}$ with $x \neq x_{\mathrm{o}}$. We define $y_{p}=-\mathscr{A} x+y^{\eta}$, so that $\left\|y_{p}\right\| \mathscr{Y} \leqslant \eta$ since $x \in \mathscr{M}$. Then, using Proposition 3.5 .

$$
\begin{aligned}
\frac{1}{2}\left(\|x\|_{\mathscr{X}}^{2}-\left\|x_{\mathrm{o}}\right\|_{\mathscr{X}}^{2}\right)=\frac{1}{2}\|x\|_{\mathscr{X}}^{2}+\mathscr{J}\left(y_{\mathrm{o}}\right)=\frac{1}{2}\|x\|_{\mathscr{X}}^{2}+\frac{1}{2}\left\|\mathscr{A}^{*} y_{\mathrm{o}}\right\|_{\mathscr{X}}^{2}+\eta\left\|y_{\mathrm{o}}\right\| \mathscr{Y}-\left(y_{\mathrm{o}}, y^{\eta}\right)_{\mathscr{Y}} \\
=\frac{1}{2}\|x\|_{\mathscr{X}}^{2}+\frac{1}{2}\left\|x_{\mathrm{o}}\right\|_{\mathscr{X}}^{2}+\eta\left\|y_{\mathrm{o}}\right\| \mathscr{Y}-\left(y_{\mathrm{o}}, \mathscr{A} x+y_{p}\right)_{\mathscr{Y}} \\
=\underbrace{\frac{1}{2}\|x\|_{\mathscr{X}}^{2}+\frac{1}{2}\left\|x_{\mathrm{o}}\right\|_{\mathscr{X}}^{2}-\left(\mathscr{A}^{*} y_{\mathrm{o}}, x\right) \mathscr{X}}_{=\frac{1}{2}\left\|x-x_{\mathrm{o}}\right\|_{\mathscr{X}}^{2}>0}+\underbrace{\eta\left\|y_{\mathrm{o}}\right\| \mathscr{Y}-\left(y_{\mathrm{o}}, y_{p}\right) \mathscr{Y}}_{\geqslant 0},
\end{aligned}
$$

which ends the proof.

As an immediate consequence, since $x_{\mathrm{s}} \in \mathscr{M}$, we obtain

Corollary 3.7. For all $\eta>0$, we have $\left\|x_{\mathrm{o}}\right\|_{\mathscr{X}} \leqslant\left\|x_{\mathrm{s}}\right\|_{\mathscr{X}}$. 
Convergence. We now prove that $x_{\mathrm{o}}$ converges to $x_{\mathrm{s}}$ as $\eta$ goes to zero. Note however that we cannot obtain the rate of convergence in this abstract framework without doing some extra assumptions on $y^{\eta}$, for example some source condition, which are in practice difficult if not impossible to verify. We shall come back on this in Section 6 .

Theorem 3.8. $x_{\mathrm{o}}$ converges to $x_{\mathrm{s}}$ when $\eta$ tends to zero.

Proof. Let us choose $\left(\eta_{n}\right)_{n \in \mathbb{N}}$ any sequence of strictly positive real numbers converging to zero, $y_{n}=y^{\eta_{n}}$ the corresponding noisy data verifying $\left\|y_{n}-y_{\mathrm{s}}\right\|_{\mathscr{Y}} \leqslant \eta_{n}$, and $x_{\mathrm{o}, \mathrm{n}}=\mathscr{A}^{*} y_{\mathrm{o}, \mathrm{n}}$ with $y_{\mathrm{o}, \mathrm{n}}$ the minimizer of the functional

$$
\mathscr{J}_{n}: y \in \mathscr{Y} \longmapsto \frac{1}{2}\left\|\mathscr{A}^{*} y\right\|_{\mathscr{X}}^{2}+\eta_{n}\|y\|_{\mathscr{Y}}-\left(y, y_{n}\right)_{\mathscr{Y}} .
$$

We have seen that the sequence $\left(x_{\mathrm{o}, \mathrm{n}}\right)_{n \in \mathbb{N}}$ is bounded by Corollary 3.7

$$
\left\|x_{\mathrm{o}, \mathrm{n}}\right\|_{\mathscr{X}} \leqslant\left\|x_{\mathrm{s}}\right\|_{\mathscr{X}} .
$$

Therefore, up to a subsequence it weakly converges to some $x_{\infty}$ belonging to $\mathscr{X}$. But, using (3.5),

$$
\left\|\mathscr{A} x_{\mathrm{o}, \mathrm{n}}-y_{\mathrm{s}}\right\|_{\mathscr{Y}} \leqslant\left\|\mathscr{A} x_{\mathrm{o}, \mathrm{n}}-y_{n}\right\|_{\mathscr{Y}}+\left\|y_{n}-y_{\mathrm{s}}\right\|_{\mathscr{Y}} \leqslant 2 \eta_{n},
$$

and then $\mathscr{A} x_{\mathrm{o}, \mathrm{n}}$ strongly converges to $y_{\mathrm{s}}$ in $\mathscr{Y}$, while it weakly converges to $\mathscr{A} x_{\infty}$, therefore $\mathscr{A} x_{\infty}=y_{\mathrm{s}}$, leading to $x_{\infty}=x_{\mathrm{s}}$. As for all $n$,

$$
\left\|x_{\mathrm{o}, \mathrm{n}}\right\|_{\mathscr{X}} \leqslant\left\|x_{\mathrm{s}}\right\|_{\mathscr{X}} \leqslant \liminf \left\|x_{\mathrm{o}, \mathrm{n}}\right\|_{\mathscr{X}},
$$

we deduce

$$
\lim _{n \rightarrow \infty}\left\|x_{\mathrm{o}, \mathrm{n}}\right\| \mathscr{X}=\left\|x_{\mathrm{s}}\right\|_{\mathscr{X}},
$$

and obtain the strong converges of the subsequence to $x_{\mathrm{s}}$. The result follows, as this reasoning is correct for any sequence of strictly positive real numbers $\left(\eta_{n}\right)_{n \in \mathbb{N}}$ converging to zero.

Remark 3.9. Note that if we do not have any rate of convergence for the method, we nevertheless know that

$$
\left\|A x_{\mathrm{o}}-y_{\mathrm{s}}\right\| \leqslant 2 \eta,
$$

i.e. we have a linear rate of convergence for the residual.

\subsection{Link with the Tikhonov regularization}

A commun way to regularize our main problem is the Tikhonov regularization, which in our context reads: for $\varepsilon>0$,

$$
x_{\varepsilon}=\underset{x \in \mathscr{X}}{\arg \min } \frac{1}{2}\left\|\mathscr{A} x-y^{\eta}\right\|_{\mathscr{Y}}^{2}+\frac{\varepsilon}{2}\|x\|_{\mathscr{X}}^{2} .
$$

It is well-known (see, among others, [30]) that such problem is well-posed, and in the case of exact data (i.e. $y^{\eta}=y_{\mathrm{s}}$ ), $x_{\varepsilon}$ converges to $x_{\mathrm{s}}$ when $\varepsilon$ goes to zero.

Furthermore, for $y^{\eta}$ such that $\left\|y-y^{\eta}\right\|_{\mathscr{Y}} \leqslant \eta<\left\|y^{\eta}\right\|_{\mathscr{Y}}$, there exists a unique value of the parameter of regularization $\varepsilon=\varepsilon(\eta)$ such that the corresponding minimizer $x_{\varepsilon}$ satisfies the Morozov discrepancy principle $\left\|\mathscr{A} x_{\varepsilon}-y^{\eta}\right\|_{\mathscr{Y}}=\eta$, automatically ensuring both stability of the reconstruction procedure and convergence towards the exact solution as $\eta$ goes to zero. This is why this parameter of regularization is often chosen in Tikhonov regularization.

It turns out that the method described above allows to automatically determine $\varepsilon(\eta)$. Indeed, it can be explicitly expressed in terms of $\eta$ and $\left\|y_{\mathrm{o}}\right\|_{\mathscr{Y}}$, whereas the corresponding $x_{\varepsilon}$ is precisely $x_{\mathrm{o}}$ (see Theorem 3.10 below). 
Theorem 3.10. For all $\eta>0$ and $y^{\eta} \in \mathscr{Y}$ such that $\left\|y_{\mathrm{s}}-y^{\eta}\right\|_{\mathscr{Y}} \leqslant \eta<\left\|y^{\eta}\right\| \mathscr{Y}$, one has

$$
\varepsilon(\eta)=\frac{\eta}{\left\|y_{\mathrm{o}}\right\|_{\mathscr{Y}}} \quad \text { and } \quad x_{\varepsilon(\eta)}=x_{\mathrm{o}}
$$

Proof. Clearly, $x_{\varepsilon}$ satisfies 3.6 if and only if for all $x \in \mathscr{X}$,

$$
\left(\mathscr{A} x_{\varepsilon}, \mathscr{A} x\right)_{\mathscr{Y}}+\varepsilon\left(x_{\varepsilon}, x\right)_{\mathscr{X}}=\left(y^{\eta}, \mathscr{A} x\right)_{\mathscr{Y}} .
$$

Now, Proposition 3.4 implies that for all $y \in \mathscr{Y}$, one has

$$
\left(\mathscr{A} \mathscr{A}^{*} y_{\mathrm{o}}, y\right)_{\mathscr{Y}}+\frac{\eta}{\left\|y_{\mathrm{o}}\right\|_{\mathscr{Y}}}\left(y_{\mathrm{o}}, y\right)_{\mathscr{Y}}=\left(y^{\eta}, y\right)_{\mathscr{Y}}
$$

which, recalling that $\mathscr{A}^{*} y_{\mathrm{o}}=x_{\mathrm{o}}$ and choosing $y=\mathscr{A} x$ for $x \in \mathscr{X}$, leads to

$$
\begin{aligned}
\left(y^{\eta}, \mathscr{A} x\right)_{\mathscr{Y}} & =\left(\mathscr{A}_{\mathscr{A}^{*}} y_{\mathrm{o}}, \mathscr{A} x\right)_{\mathscr{Y}}+\frac{\eta}{\left\|y_{\mathrm{o}}\right\|_{\mathscr{Y}}}\left(y_{\mathrm{o}}, \mathscr{A} x\right)_{\mathscr{Y}} \\
& =\left(\mathscr{A} x_{\mathrm{o}}, \mathscr{A} x\right)_{\mathscr{Y}}+\frac{\eta}{\left\|y_{\mathrm{o}}\right\| \mathscr{Y}}\left(\mathscr{A}^{*} y_{\mathrm{o}}, x\right)_{\mathscr{X}} \\
& =\left(\mathscr{A} x_{\mathrm{o}}, \mathscr{A} x\right)_{\mathscr{Y}}+\frac{\eta}{\left\|y_{\mathrm{o}}\right\| \mathscr{Y}}\left(x_{\mathrm{o}}, x\right)_{\mathscr{X}} .
\end{aligned}
$$

Therefore, $x_{\mathrm{o}}$ is the solution of 3.6 associated to the parameter choice $\varepsilon=\frac{\eta}{\left\|y_{\mathrm{o}}\right\|_{\mathscr{O}}}$. The fact that this parameter is such that the corresponding minimizer satisfies the Morozov discrepancy principle follows from equation (3.5), which ends the proof.

\section{Application to the data completion problem}

We are now in position to prove all the results announced in the introduction, that is Theorem 1.4 . Theorem 1.5, Theorem 1.6 and Theorem 1.10, using the results of Section 3 in the functional setting defined in Appendix A, that is with $\mathscr{X}=\mathrm{H}^{-1 / 2}\left(\Gamma_{\mathrm{c}}\right) \times \mathrm{H}_{\diamond}^{1 / 2}\left(\Gamma_{\mathrm{c}}\right)$ defined in Section A.1, $\mathscr{Y}=\mathbf{H}(\Omega)$ defined in Section A.2, and the operator $\mathscr{A}=A$ defined in Section 2 . Notice also that $\eta=c \delta$ with $c$ and $\delta$ being defined in Section 1 reads

Using Proposition 2.2, we obtain that the functional $\mathscr{J}$ defined by (3.1), that we want to minimize,

$$
\mathscr{J}: \boldsymbol{p} \in \mathbf{H} \longmapsto \int_{\Omega}\left(\left|\nabla v_{\varphi_{p}}\right|^{2}+\left|\nabla v_{\psi_{p}}\right|^{2}\right) \mathrm{d} x+c \delta\left\|\nabla w_{p}\right\|_{\mathbf{L}^{2}(\Omega)}-\int_{\Omega} \boldsymbol{F}^{\delta} \cdot \nabla w_{p} \mathrm{~d} x,
$$

where $w_{p}$ is any harmonic $\mathrm{H}^{1}$-function so that $\nabla w_{p}=\boldsymbol{p}$, and $v_{\varphi_{p}}$ and $v_{\psi_{p}}$ are defined by (1.7), with

$$
\varphi_{p}=\partial_{\nu} w_{p \mid \Gamma_{\mathrm{c}}} \quad \text { and } \quad \psi_{p}=-\left(w_{p \mid \Gamma_{\mathrm{c}}}-\frac{1}{\left|\Gamma_{\mathrm{c}}\right|} \int_{\Gamma_{\mathrm{c}}} w_{p} \mathrm{~d} s\right) .
$$

Following the results of the previous section (see 3.2 ), we define $\boldsymbol{p}_{\mathrm{o}} \in \mathbf{H}(\Omega)$ as the unique minimizer of $\mathscr{J}$,

$$
p_{\mathrm{o}}=\underset{\boldsymbol{p} \in \mathbf{H}(\Omega)}{\arg \min } \mathscr{J}(\boldsymbol{p}),
$$

and our regularized solution (see 3.3 )

$$
\left(\varphi_{\mathrm{o}}, \psi_{\mathrm{o}}\right)=A^{*} \boldsymbol{p}_{\mathrm{o}}=\left(\partial_{\nu} w_{p_{\mathrm{o}} \mid \Gamma_{\mathrm{c}}},-w_{p_{\mathrm{o}} \mid \Gamma_{\mathrm{c}}}+\frac{1}{\left|\Gamma_{\mathrm{c}}\right|} \int_{\Gamma_{\mathrm{c}}} w_{p_{\mathrm{o}}} \mathrm{d} s\right),
$$

where again $w_{p_{\mathrm{o}}}$ is any harmonic $\mathrm{H}^{1}$ function so that $\nabla w_{p_{\mathrm{o}}}=\boldsymbol{p}_{\mathrm{o}}$. 
Reparametrization: proofs of Theorem 1.4 and Theorem 1.5. Numerically, handling the space $\mathbf{H}(\Omega)$ might be complicated, in particular because of the harmonicity condition. Therefore, we reparametrize $\mathbf{H}(\Omega)$ through boundary conditions as follows. First of all, we recall (see Section 1 that, for any $\theta \in \mathrm{H}_{\diamond}^{-1 / 2}(\partial \Omega)$, with

$$
\mathrm{H}_{\diamond}^{-1 / 2}(\partial \Omega)=\left\{\theta \in \mathrm{H}^{-1 / 2}(\partial \Omega),\langle\theta, 1\rangle=0\right\},
$$

we denote $w(\theta)$ the function of $\mathrm{H}^{1}(\Omega)$ verifying $\int_{\Gamma_{\mathrm{c}}} w(\theta) \mathrm{d} s=0$ and

$$
\left\{\begin{array}{rlll}
\Delta w(\theta) & = & & \text { in } \Omega \\
\partial_{\nu} w(\theta) & = & & \text { on } \partial \Omega
\end{array}\right.
$$

Note that the application $\theta \in \mathrm{H}_{\diamond}^{-1 / 2}(\partial \Omega) \longmapsto \nabla w(\theta) \in \mathbf{L}^{2}(\Omega)$ is linear.

We have the following lemma.

Lemma 4.1. For all $\boldsymbol{p} \in \mathbf{H}(\Omega)$, there exists a unique $\theta \in \mathrm{H}_{\diamond}^{-1 / 2}(\partial \Omega)$ such that $\nabla w(\theta)=\boldsymbol{p}$, where $w(\theta) \in \mathrm{H}^{1}(\Omega)$ is defined above.

Proof. Let us begin by proving the existence. Let $\boldsymbol{p} \in \mathbf{H}(\Omega)$. By definition, there exists $W \in \mathrm{H}^{1}(\Omega)$ such that $\Delta W=0$ and $\nabla W=\boldsymbol{p}$. For any $v \in \mathrm{H}^{1}(\Omega)$, one has

$$
\left\langle\partial_{\nu} W, v\right\rangle=\int_{\Omega} \nabla W \cdot \nabla v \mathrm{~d} x
$$

which shows that $\partial_{\nu} W \in \mathrm{H}_{\diamond}^{-1 / 2}(\partial \Omega)$ choosing $v=1$. Hence clearly $\boldsymbol{p}=\nabla w\left(\partial_{\nu} \tilde{W}\right)$, where

$$
\tilde{W}=W-\frac{1}{\left|\Gamma_{\mathrm{c}}\right|} \int_{\Gamma_{\mathrm{c}}} W \mathrm{~d} s
$$

Now let us prove the uniqueness. Let $\theta_{1}, \theta_{2} \in \mathrm{H}_{\diamond}^{-1 / 2}(\partial \Omega)$ such that $\boldsymbol{p}=\nabla w\left(\theta_{1}\right)=\nabla w\left(\theta_{2}\right)$. Then by definition one has, for all $v \in \mathrm{H}^{1}(\Omega)$,

$$
\left\langle\theta_{1}, v\right\rangle=\int_{\Omega} \nabla w\left(\theta_{1}\right) \cdot \nabla v \mathrm{~d} x=\int_{\Omega} \nabla w\left(\theta_{2}\right) \cdot \nabla v \mathrm{~d} x=\left\langle\theta_{2}, v\right\rangle .
$$

Hence $\theta_{1}=\theta_{2}$.

This result permits to replace the minimization problem

$$
p_{\mathrm{o}}=\underset{p \in H}{\arg \min }\left\{\mathscr{J}(\boldsymbol{p})=\frac{1}{2} \int_{\Omega}\left(\left|\nabla v_{\varphi_{p}}\right|^{2}+\left|\nabla v_{\psi_{p}}\right|^{2}\right) \mathrm{d} x+c \delta\left(\int_{\Omega}|\boldsymbol{p}|^{2} \mathrm{~d} x\right)^{\frac{1}{2}}-\int_{\Omega} \boldsymbol{F}^{\delta} \cdot \boldsymbol{p} \mathrm{d} x\right\},
$$

by a minimization problem over $\mathrm{H}_{\diamond}^{-1 / 2}(\partial \Omega)$, easier to handle numerically, which reads

$$
\theta_{\mathrm{o}}=\underset{\theta \in \mathrm{H}_{\diamond}^{-1 / 2}(\partial \Omega)}{\arg \min }\left\{\mathscr{F}(\theta)=\frac{1}{2} \int_{\Omega}\left(\left|\nabla v_{1}\right|^{2}+\left|\nabla v_{2}\right|^{2}\right) \mathrm{d} x+c \delta\left(\int_{\Omega}|\nabla w(\theta)|^{2} \mathrm{~d} x\right)^{\frac{1}{2}}-\int_{\Omega} \boldsymbol{F}^{\delta} \cdot \nabla w(\theta) \mathrm{d} x\right\},
$$

with $v_{1}$ and $v_{2}$ being two harmonic functions in $\mathrm{H}^{1}(\Omega)$ such that

$$
v_{1 \mid \Gamma}=0, \quad \partial_{\nu} v_{1 \mid \Gamma_{\mathrm{c}}}=\theta, \quad \partial_{\nu} v_{2 \mid \Gamma}=0 \quad \text { and } \quad v_{2 \mid \Gamma_{\mathrm{c}}}=w(\theta)_{\mid \Gamma_{\mathrm{c}}} .
$$


Then we have

$$
\boldsymbol{p}_{\mathrm{o}}=\nabla w\left(\theta_{\mathrm{o}}\right),
$$

and we use the fact that $\boldsymbol{p}_{\mathrm{o}}$ is the unique solution of 4.1) and Lemma 4.1 to prove Theorem 1.4 i.e. there exists a unique minimizer $\theta_{\mathrm{o}} \in \mathrm{H}_{\diamond}^{-1 / 2}(\partial \Omega)$ of $\mathscr{F}$.

Moreover, in our context, Equation 3.5 reads

$$
\left\|A\left(\varphi_{\mathrm{o}}, \psi_{\mathrm{o}}\right)-\boldsymbol{F}^{\delta}\right\|_{\mathbf{L}^{2}(\Omega)}=c \delta \Longleftrightarrow\left\|\nabla v_{\varphi_{\mathrm{o}}}-\nabla v_{\psi_{\mathrm{o}}}-\boldsymbol{F}^{\delta}\right\|_{\mathbf{L}^{2}(\Omega)}=c \delta
$$

that is, taking into account of the expression of $\left(\varphi_{\mathrm{o}}, \psi_{\mathrm{o}}\right)$ with respect to $w_{p_{\mathrm{o}}}$ (see 4.2) and since $\boldsymbol{p}_{\mathrm{o}}=\nabla w\left(\theta_{\mathrm{o}}\right)$,

$$
\left\|\nabla v_{1}\left(\theta_{\mathrm{o}}\right)-\nabla v_{2}\left(\theta_{\mathrm{o}}\right)-\boldsymbol{F}^{\delta}\right\|_{\mathbf{L}^{2}(\Omega)}=c \delta
$$

which proves Theorem 1.5

Convergence: proof of Theorem 1.6. We recall that $\left(\varphi_{\mathrm{ex}}, \psi_{\mathrm{ex}}\right)$ denotes the exact missing data associated to the exact solution $u_{\text {ex }}$ (see Section 1). We now state the two following results which proves Theorem 1.6 .

Proposition 4.2. The couple $\left(\varphi_{\mathrm{o}}, \psi_{\mathrm{o}}\right)$ converges to $\left(\varphi_{\mathrm{ex}}, \psi_{\mathrm{ex}}\right)$ strongly in $\mathrm{H}^{-1 / 2}\left(\Gamma_{\mathrm{c}}\right) \times \tilde{\mathrm{H}}^{1 / 2}\left(\Gamma_{\mathrm{c}}\right)$ as $\delta$ goes to zero.

Proof. Suppose that we have proven that

$$
A\left(\varphi_{\mathrm{ex}}, \tilde{\psi}_{\mathrm{ex}}\right)=\boldsymbol{F}=\nabla u_{\mathrm{N}}-\nabla u_{\mathrm{D}},
$$

where $u_{\mathrm{N}}$ and $u_{\mathrm{D}}$ are defined in 1.2 and where

$$
\tilde{\psi}_{\mathrm{ex}}=\psi_{\mathrm{ex}}-\frac{1}{\left|\Gamma_{\mathrm{c}}\right|} \int_{\Gamma_{\mathrm{c}}} \psi_{\mathrm{ex}} \mathrm{d} s \in \mathrm{H}_{\diamond}^{1 / 2}\left(\Gamma_{\mathrm{c}}\right) .
$$

Then Theorem 3.8 directly implies the convergence of $\left(\varphi_{\mathrm{o}}, \psi_{\mathrm{o}}\right)$ to $\left(\varphi_{\mathrm{ex}}, \tilde{\psi}_{\mathrm{ex}}\right)$, which in turn implies the result as $\tilde{\psi}_{\text {ex }}=\psi_{\text {ex }}$ in $\tilde{\mathrm{H}}^{1 / 2}\left(\Gamma_{\mathrm{c}}\right)$.

Remains to prove (4.4). We first note that

$$
A\left(\varphi_{\mathrm{ex}}, \tilde{\psi}_{\mathrm{ex}}\right)=\nabla v_{\varphi_{\mathrm{ex}}}-\nabla v_{\tilde{\psi}_{\mathrm{ex}}}=\nabla v_{\varphi_{\mathrm{ex}}}-\nabla v_{\psi_{\mathrm{ex}}},
$$

as by construction $v_{\tilde{\psi}_{\mathrm{ex}}}=v_{\psi_{\mathrm{ex}}}+\alpha$ for some real parameter $\alpha$. Then 4.4 is equivalent to

$$
\nabla\left(v_{\varphi_{\mathrm{ex}}}+u_{\mathrm{D}}\right)=\nabla\left(v_{\psi_{\mathrm{ex}}}+u_{\mathrm{N}}\right) .
$$

But this last equation is necessarily true, as it is not difficult to see from the problem they solve that $v_{\varphi_{\mathrm{ex}}}+u_{\mathrm{D}}=u_{\mathrm{ex}}=v_{\psi_{\mathrm{ex}}}+u_{\mathrm{N}}$.

Corollary 4.3. The function $u_{\mathrm{o}}$, defined by 1.6 , converges to $u_{\mathrm{ex}}$ strongly in $\mathrm{H}^{1}(\Omega)$, as $\delta$ goes to zero.

Proof. This is direct consequence of the previous proposition, as $u_{\mathrm{o}}-u_{\mathrm{ex}}$ satisfies $\Delta\left(u_{\mathrm{o}}-u_{\mathrm{ex}}\right)=0$ in $\Omega$, $u_{\mathrm{o}}-u_{\mathrm{ex}}=g_{\mathrm{D}}^{\delta}-g_{\mathrm{D}}$ on $\Gamma$ and $\partial_{\nu}\left(u_{\mathrm{o}}-u_{\mathrm{ex}}\right)=\varphi_{\mathrm{o}}-\varphi_{\mathrm{ex}}$ on $\Gamma_{\mathrm{c}}$. 
Link with the Kohn-Vogelius regularization: proof of Theorem 1.10. We now focus on the Tikhonov regularization of the Cauchy problem, which is based on the minimization problem (3.6). In our context, for $\varepsilon>0$, the quadratic functional to minimize turns out to be

$$
(\varphi, \psi) \in \mathrm{H}^{-1 / 2}\left(\Gamma_{\mathrm{c}}\right) \times \mathrm{H}_{\diamond}^{1 / 2}\left(\Gamma_{\mathrm{c}}\right) \longmapsto \frac{1}{2} \int_{\Omega}\left|\nabla v_{\varphi}-\nabla v_{\psi}-\boldsymbol{F}^{\delta}\right|^{2} \mathrm{~d} x+\frac{\varepsilon}{2} \int_{\Omega}\left(\left|\nabla v_{\varphi}\right|^{2}+\left|\nabla v_{\psi}\right|^{2}\right) \mathrm{d} x,
$$

that is precisely the Kohn-Vogelius functional used in 21] to regularize the data completion problem. Hence Theorem 1.10 is a direct consequence Theorem 3.10 up to the reparametrization of our minimization problem discussed above.

\section{$5 \quad$ Numerical simulations}

\subsection{Context for the numerical simulations}

As mentioned previously in Remark 1.3 in order to numerically solve our problem, it is mandatory to know an approximation of a constant $c$ such that (see 1.3 )

$$
\left\|\boldsymbol{F}^{\delta}-\boldsymbol{F}\right\|_{\mathbf{L}^{2}(\Omega)} \leqslant c \delta
$$

This question is nontrivial as $c$ depends on Poincaré constants and trace constants, both of them being difficult to estimate theoretically. Therefore, we follow a naive numerical strategy in order to estimate it. More precisely, for a given data $g_{\mathrm{D}}$, we construct the corresponding $g_{\mathrm{N}}$, and then compute $u_{\mathrm{D}}$ and $u_{\mathrm{N}}$, and finally $\boldsymbol{F}=\nabla u_{\mathrm{N}}-\nabla u_{\mathrm{D}}$. Doing so for several Cauchy pair $\left(g_{\mathrm{D}}^{n}, g_{\mathrm{N}}^{n}\right)$, for $n=1, \ldots, N$, with $N \in \mathbb{N}$, we define

$$
c=\max _{n=1, \ldots, N} \frac{\left\|\boldsymbol{F}^{n}\right\|_{\mathbf{L}^{2}(\Omega)}}{\left\|g_{\mathrm{N}}^{n}\right\|_{\mathrm{H}^{-1 / 2}(\Gamma)}+\left\|g_{\mathrm{D}}^{n}\right\|_{\mathrm{H}^{1 / 2}(\Gamma)}} .
$$

Note that by definition this $c$ is actually smaller than the correct constant.

We perform this for the following dataset

$$
g_{\mathrm{D}}=\cos (k \theta), \quad g_{\mathrm{D}}=\sin (k \theta) \quad \text { and } \quad g_{\mathrm{D}}=x \mathrm{e}^{x+y}+y^{3}+\cos (x),
$$

with $k=1, \ldots, 100$, and where $\theta$ is the polar angle. Then, for the square and the annulus used in the simulations done in Section 5.2 we find respectively $c=0.402361$ and $c=0.412202$, and for the cube used in the simulations done in Section 5.3. we find $c=0.779726$. Thus, in the below simulations, we choose $c=1$.

Remark 5.1. To be very precise, in order to compute the constante $c$, we use for numerical simplicity $\left\|g_{\mathrm{N}}\right\|_{\mathrm{L}^{2}(\Gamma)}+\left\|g_{\mathrm{D}}\right\|_{\mathrm{L}^{2}(\Gamma)}$ instead of $\left\|g_{\mathrm{N}}\right\|_{\mathrm{H}^{-1 / 2}(\Gamma)}+\left\|g_{\mathrm{D}}\right\|_{\mathrm{H}^{1 / 2}(\Gamma)}$. It is of course possible to obtain numerical approximations of the norms $\|\cdot\|_{\mathrm{H}^{-1 / 2}(\Gamma)}$ and $\|\cdot\|_{\mathrm{H}^{1 / 2}(\Gamma)}$, as in [6], but it becomes costly for a result that we believe would be close to the one we obtain.

In order to solve the initial Cauchy problem (1.1), taking into account of the duality strategy exposed above, we recall that we want to find

$$
\theta_{\mathrm{O}}=\underset{\theta \in \mathrm{H}_{\diamond}^{-1 / 2}(\partial \Omega)}{\arg \min }\left\{\mathscr{F}(\theta)=\frac{1}{2} \int_{\Omega}\left(\left|\nabla v_{1}\right|^{2}+\left|\nabla v_{2}\right|^{2}\right) \mathrm{d} x+\delta\left(\int_{\Omega}|\nabla w(\theta)|^{2} \mathrm{~d} x\right)^{\frac{1}{2}}-\int_{\Omega} \boldsymbol{F}^{\delta} \cdot \nabla w(\theta) \mathrm{d} x\right\},
$$

with $v_{1}$ and $v_{2}$ being two harmonic functions in $\mathrm{H}^{1}(\Omega)$ such that

$$
v_{1 \mid \Gamma}=0, \quad \partial_{\nu} v_{1 \mid \Gamma_{\mathrm{c}}}=\theta, \quad \partial_{\nu} v_{2 \mid \Gamma}=0 \quad \text { and } \quad v_{2 \mid \Gamma_{\mathrm{c}}}=w(\theta)_{\mid \Gamma_{\mathrm{c}}},
$$


where $w(\theta) \in \mathrm{H}^{1}(\Omega)$ is the solution of

$$
\int_{\Omega} \nabla w(\theta) \cdot \nabla v \mathrm{~d} x=\langle\theta, v\rangle, \quad \forall v \in \mathrm{H}^{1}(\Omega), \quad \text { with } \quad \int_{\Gamma_{c}} w(\theta) \mathrm{d} s=0,
$$

and where $\boldsymbol{F}^{\delta}=\nabla u_{\mathrm{N}}^{\delta}-\nabla u_{\mathrm{D}}^{\delta}$ with $u_{\mathrm{D}}^{\delta}$ and $u_{\mathrm{N}}^{\delta}$ in $\mathrm{H}^{1}(\Omega)$ being the unique harmonic functions satisfying the following limit conditions:

$$
u_{\mathrm{D} \mid \Gamma}=g_{\mathrm{D}}^{\delta}, \quad \partial_{\nu} u_{\mathrm{D} \mid \Gamma_{\mathrm{c}}}=0, \quad \partial_{\nu} u_{\mathrm{N} \mid \Gamma}=g_{\mathrm{N}}^{\delta} \quad \text { and } \quad u_{\mathrm{N} \mid \Gamma_{\mathrm{c}}}=0 .
$$

Then, according to Section 4 , the solution of the dual problem is given by $\boldsymbol{p}_{\mathrm{o}}=\nabla w\left(\theta_{\mathrm{o}}\right)($ see $4.3 \mathrm{p})$ and our regularization solution is given by (see 4.2p)

$$
\left(\varphi_{\mathrm{o}}, \psi_{\mathrm{o}}\right)=A^{*} \boldsymbol{p}_{\mathrm{o}}=\left(\partial_{\nu} w\left(\theta_{\mathrm{o}}\right)_{\mid \Gamma_{\mathrm{c}}},-w\left(\theta_{\mathrm{o}}\right)_{\mid \Gamma_{\mathrm{c}}}\right) .
$$

In order to numerically solve the above optimization problem, we use a classical gradient method. Let $\tilde{\theta} \in \mathrm{H}_{\diamond}^{-1 / 2}(\partial \Omega)$. We easily compute:

$$
\begin{aligned}
\nabla \mathscr{F}(\theta) \cdot \tilde{\theta}=\int_{\Omega}\left(\nabla v_{1}(\theta) \cdot \nabla v_{1}(\tilde{\theta})+\nabla v_{2}(\theta) \cdot \nabla v_{2}(\tilde{\theta})\right) \mathrm{d} x \\
\quad+\frac{\delta}{\|\nabla w(\theta)\|_{\mathbf{L}^{2}(\Omega)}} \int_{\Omega} \nabla w(\theta) \cdot \nabla w(\tilde{\theta}) \mathrm{d} x-\int_{\Omega} \boldsymbol{F}^{\delta} \cdot \nabla w(\tilde{\theta}) \mathrm{d} x .
\end{aligned}
$$

But, using Green's formula,

$$
\int_{\Omega} \nabla v_{1}(\theta) \cdot \nabla v_{1}(\tilde{\theta}) \mathrm{d} x=\left\langle\partial_{\nu} v_{1}(\tilde{\theta}), v_{1}(\theta)\right\rangle_{\partial \Omega}=\left\langle\tilde{\theta}, v_{1}(\theta)\right\rangle_{\Gamma_{\mathrm{c}}}=\left\langle\tilde{\theta}, v_{1}(\theta)\right\rangle_{\partial \Omega}
$$

and

$$
\int_{\Omega} \nabla v_{2}(\theta) \cdot \nabla v_{2}(\tilde{\theta}) \mathrm{d} x=\left\langle\partial_{\nu} v_{2}(\theta), v_{2}(\tilde{\theta})\right\rangle_{\partial \Omega}=\left\langle\partial_{\nu} v_{2}(\theta), w(\tilde{\theta})\right\rangle_{\partial \Omega}=\int_{\Omega} \nabla v_{2}(\theta) \cdot \nabla w(\tilde{\theta}) \mathrm{d} x=\left\langle\tilde{\theta}, v_{2}(\theta)\right\rangle_{\partial \Omega} .
$$

Moreover

$$
\int_{\Omega} \nabla w(\theta) \cdot \nabla w(\tilde{\theta}) \mathrm{d} x=\langle\tilde{\theta}, w(\theta)\rangle_{\partial \Omega}
$$

and

$$
\int_{\Omega} \boldsymbol{F}^{\delta} \cdot \nabla w(\tilde{\theta}) \mathrm{d} x=\left\langle\tilde{\theta},\left(u_{\mathrm{N}}^{\delta}-u_{\mathrm{D}}^{\delta}\right)\right\rangle_{\partial \Omega}
$$

Thus we obtain

$$
\nabla \mathscr{F}(\theta) \cdot \tilde{\theta}=\left\langle\tilde{\theta}, v_{1}(\theta)+v_{2}(\theta)+\frac{\delta}{\|\nabla w(\theta)\|_{\mathbf{L}^{2}(\Omega)}} w(\theta)+u_{\mathrm{N}}^{\delta}-u_{\mathrm{D}}^{\delta}\right\rangle_{\partial \Omega},
$$

and a descent direction is given by

$$
\tilde{\theta}=-v_{1}(\theta)-v_{2}(\theta)-\frac{\delta}{\|\nabla w(\theta)\|_{\mathbf{L}^{2}(\Omega)}} w(\theta)-u_{\mathrm{N}}^{\delta}+u_{\mathrm{D}}^{\delta} .
$$

We perform the following simulations using FreEFEM++ (see [33]). We detail below the data of each simulation. In order to have a suitable pair of Cauchy data, we use synthetic data: we fix a Dirichlet boundary condition $\psi^{*}$ on $\Gamma_{\mathrm{c}}$, we solve the Laplace's equation with an explicit data $g_{\mathrm{D}}$ on $\Gamma$ by means of another finite element method (here a P2b finite element discretization) from where we extract the corresponding data $g_{\mathrm{N}}$ by computing the value $\partial_{\nu} u$ on $\Gamma$. We specify that we add $1 \%$ of noise on $g_{\mathrm{D}}$ and $g_{\mathrm{N}}$ for the simulations. 


\subsection{Numerical results in the two dimensional case}

Firstly, we perform the reconstruction of boundary data in the two dimensional case. In Figure 1 . we aim at reconstructing the data on the upper boundary of the square $(-0.5,0.5)^{2}$ and we consider $g_{\mathrm{D}}=\psi^{*}=y^{3}-3 x^{2} y$. This simulation underlines the efficiency of the method. Notice also that the we obtain almost the minimum value of the functional in few iterations (see Figure 1d), even if the additional iterations permit to obtain a better approximation of the solution.

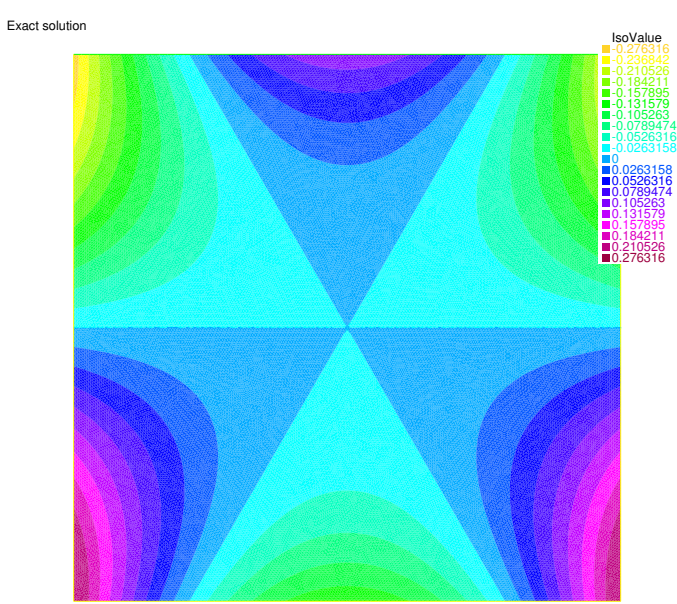

(a) Exact solution

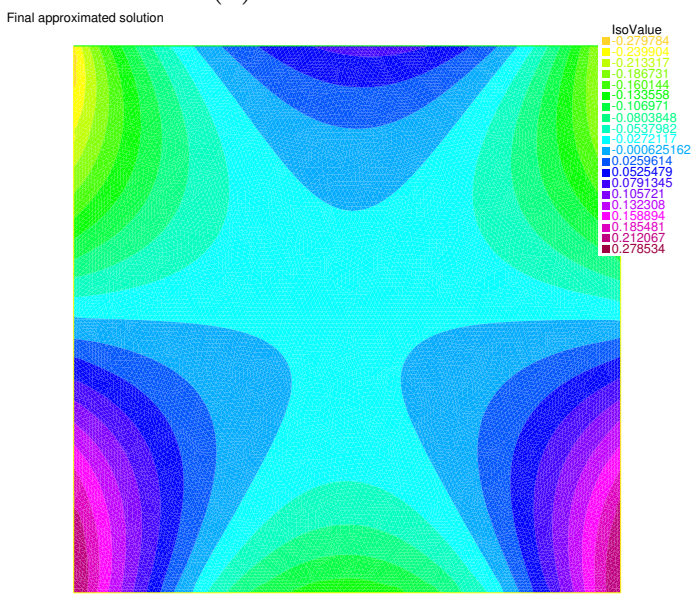

(c) Approximated solution

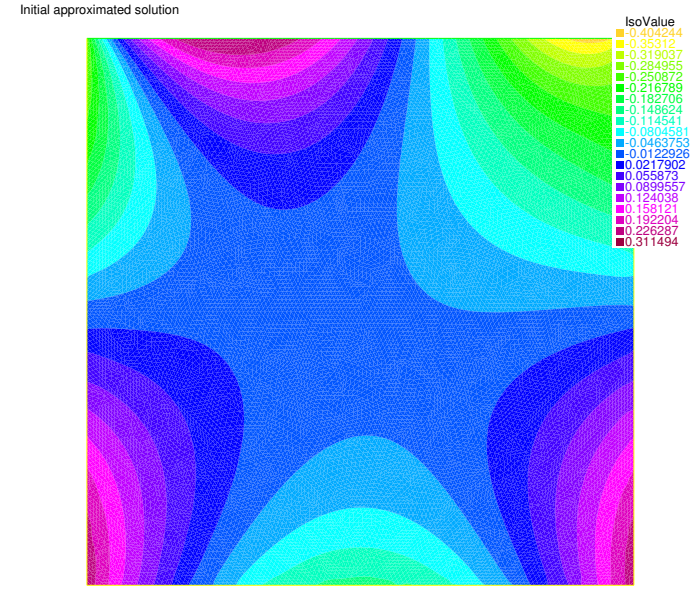

(b) Initial solution

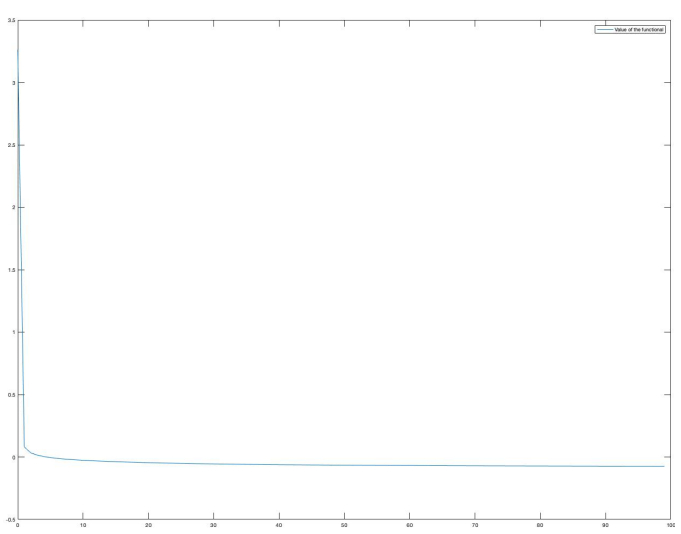

(d) Value of the functional

Figure 1: Simulations when $\Gamma_{\mathrm{c}}$ is the upper boundary of the square, with $1 \%$ of noise.

We also present in Figure 2 the same simulation (the exact solution if the same as in Figure $1 \mathrm{a}$ when we want to reconstruct the data on the upper boundary and the right boundary of the same square. Naturally, we obtain a worse approximation $u_{\mathrm{o}}$ of the solution $u_{\mathrm{ex}}$. However notice that we obtain $\left\|u_{\mathrm{ex}}-u_{\mathrm{o}}\right\|_{\mathrm{L}^{2}(\Omega)}=0.0551284,\left\|u_{\mathrm{ex}}-u_{\mathrm{o}}\right\|_{\mathrm{L}^{2}(\Gamma)}=0.182308$ and $\left\|\partial_{\nu} u_{\mathrm{ex}}-\partial_{\nu} u_{\mathrm{o}}\right\|_{\mathrm{L}^{2}(\Gamma)}=0.838961$.

Finally we consider the case of the annulus $\mathcal{C}((0,0), 1) \backslash \overline{\mathcal{C}((0,0), 0.35)}$ with the same $g_{\mathrm{D}}$ and $\psi^{*}$ than before. In Figure 3 , we assume that the unknown boundary is the boundary of the inclusion, and conversely in Figure 4. We can notice that the reconstruction is more efficient when the measurements are made on the exterior boundary. 


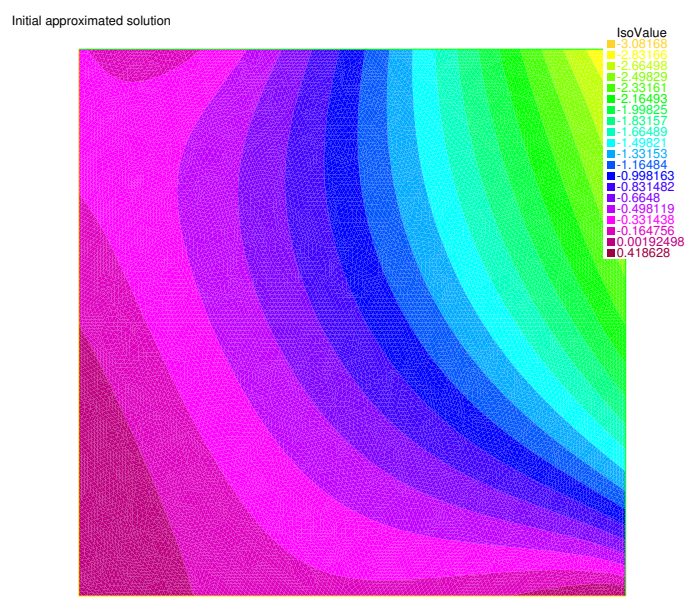

(a) Initial solution

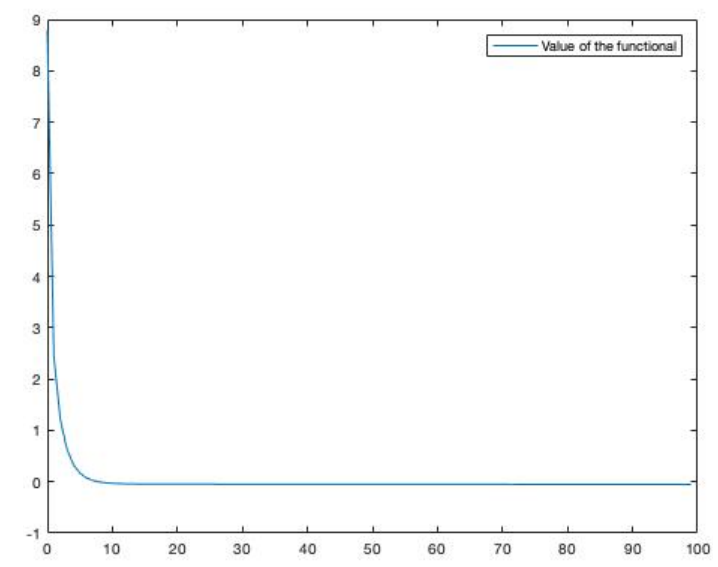

(c) Value of the functional

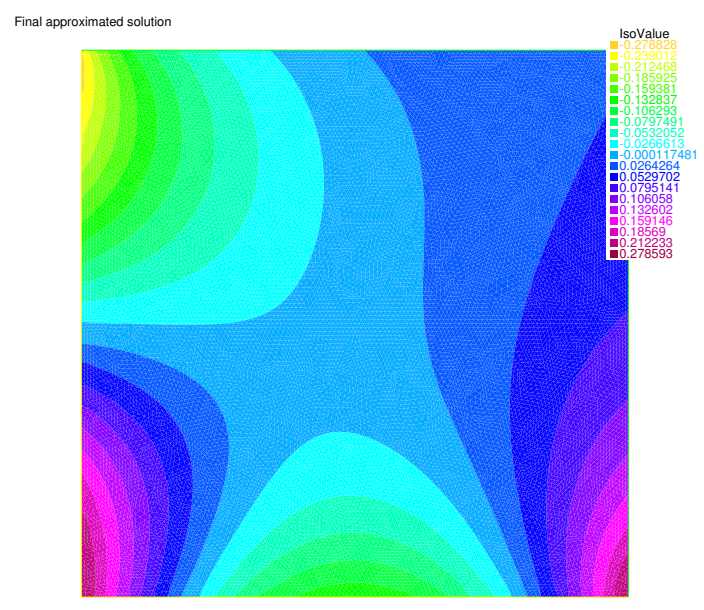

(b) Approximated solution

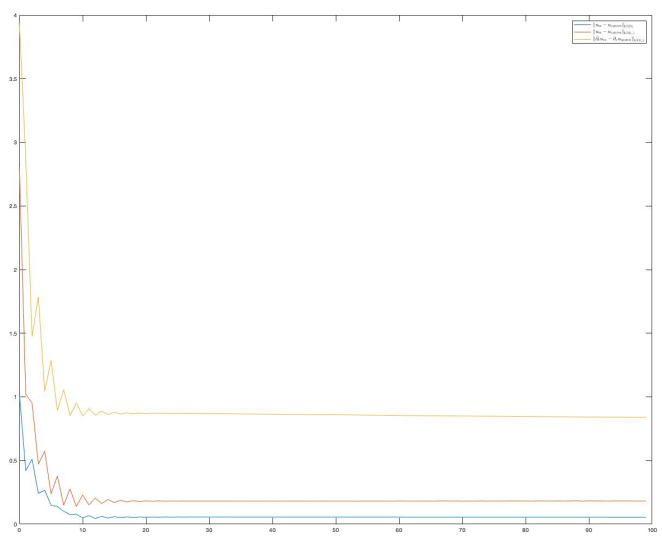

(d) Value of the error

Figure 2: Simulations when $\Gamma_{\mathrm{c}}$ is the upper and the right boundaries of the square, with $1 \%$ of noise.

\subsection{Numerical results in the three dimensional case}

To conclude these numerical simulations, we present hereafter an example of numerical reconstruction in the three dimensional case. We consider the case of the cube $(0,1)^{3}$ with $g_{\mathrm{D}}=\psi^{*}=y^{3}-3 x^{2} y+10 z$ and $\Gamma_{\mathrm{c}}$ is composed by the upper and lower sides. Once again, the value of the functional decreases until it becomes constant, and we then obtain an approximation $u_{\mathrm{o}}$ of the solution.

\section{$6 \quad$ Further comments}

\subsection{Rate of convergence of the method}

As already noted, no rate of convergence for the method is obtained in the abstract setting developed in Section 3 without additional assumption on the data. Nevertheless, in our situation, an unconditional 


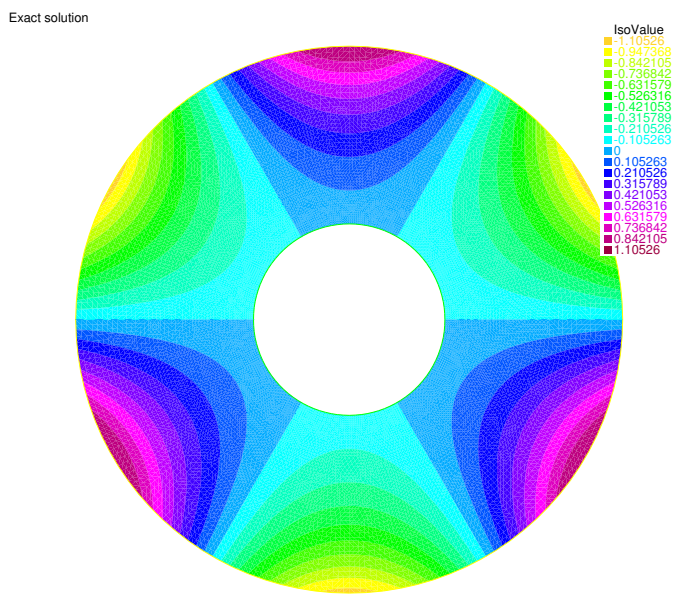

(a) Exact solution

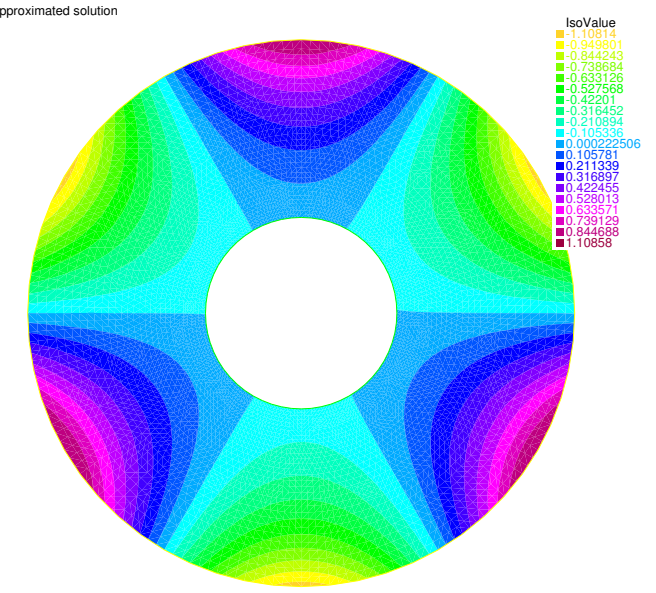

(c) Approximated solution

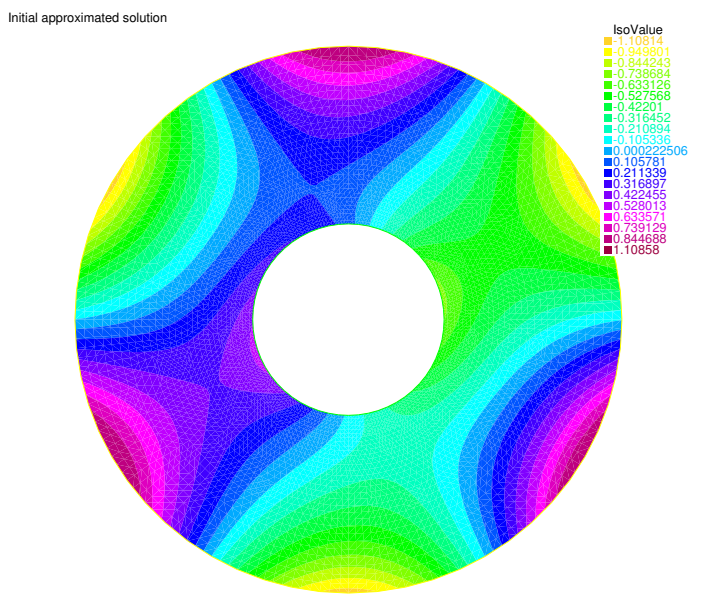

(b) Initial solution

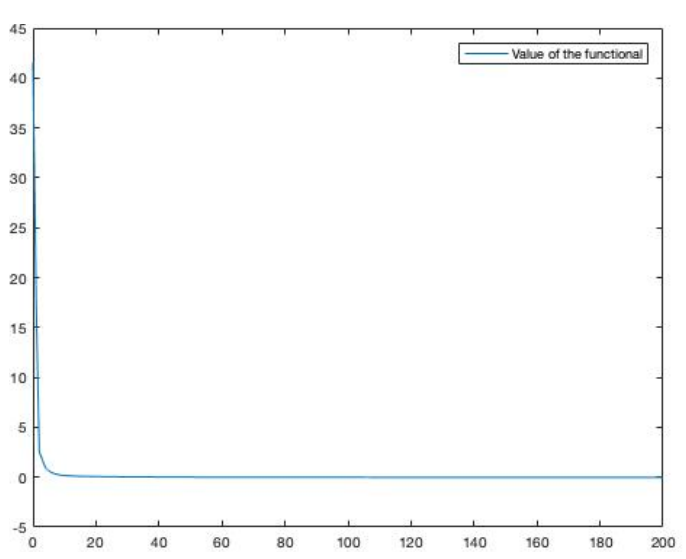

(d) Value of the functional

Figure 3: Simulations when $\Gamma_{\mathrm{c}}$ is the interior boundary, with $1 \%$ of noise.

rate of convergence can be obtained thanks to Theorem 1.1. This is another example of the link between Carleman estimates and Tikhonov regularization for partial differential equations (see, e.g., 34]).

Theorem 6.1. There exist $\delta_{0} \in(0,1], \mu \in(0,1)$ and $C>0$ such that for all $\delta \in\left(0, \delta_{0}\right)$,

$$
\left\|u_{\mathrm{o}}-u_{\mathrm{ex}}\right\|_{\mathrm{L}^{2}(\Omega)} \leqslant \frac{C}{\ln \left(\frac{C+\delta}{\delta}\right)^{\mu}} .
$$

Proof. Let $\delta \leqslant 1$. Then we have

$$
\left\|g_{\mathrm{D}}^{\delta}\right\|_{\mathrm{H}^{-1 / 2}(\Gamma)} \leqslant\left\|g_{\mathrm{D}}\right\|_{\mathrm{H}^{-1 / 2}(\Gamma)}+\delta \leqslant\left\|g_{\mathrm{D}}\right\|_{\mathrm{H}^{-1 / 2}(\Gamma)}+1 .
$$

Moreover, by Lemma A.3 and Corollary 3.7, there exists a constant $C>0$ such that

$$
\left\|\varphi_{\mathrm{o}}\right\|_{\mathrm{H}^{-1 / 2}\left(\Gamma_{\mathrm{c}}\right)}^{2} \leqslant C \int_{\Omega}\left(\left|\nabla v_{\varphi_{\mathrm{o}}}\right|^{2}+\left|\nabla v_{\psi_{\mathrm{o}}}\right|^{2}\right) \mathrm{d} x \leqslant C \int_{\Omega}\left(\left|\nabla v_{\varphi_{\mathrm{ex}}}\right|^{2}+\left|\nabla v_{\psi_{\mathrm{ex}}}\right|^{2}\right) \mathrm{d} x .
$$

Then, since $u_{\mathrm{o}}$ solves $(1.6)$, there exists a positive constant, still denoted by $C$, so that, for all $\delta \in(0,1)$,

$$
\left\|u_{\mathrm{o}}-u_{\mathrm{ex}}\right\|_{\mathrm{H}^{1}(\Omega)} \leqslant C \text {. }
$$




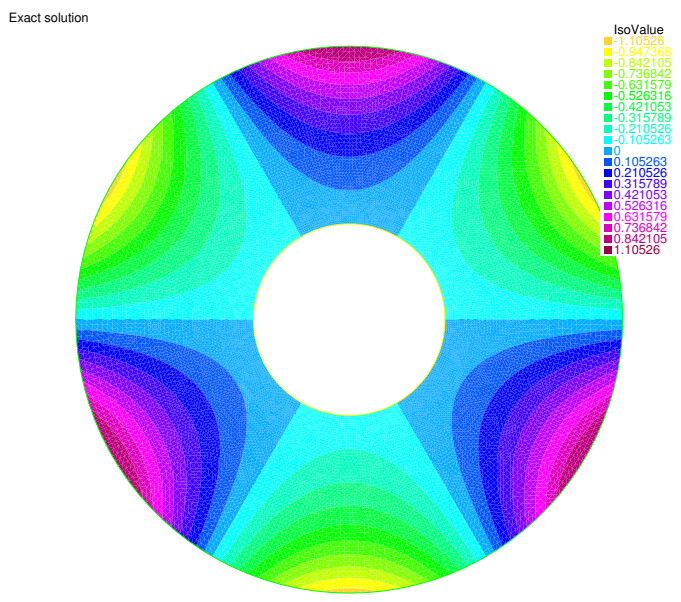

(a) Exact solution

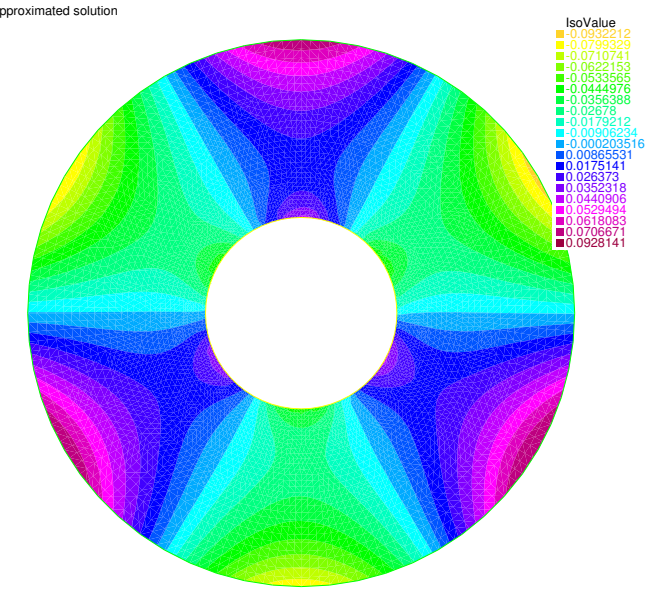

(c) Approximated solution

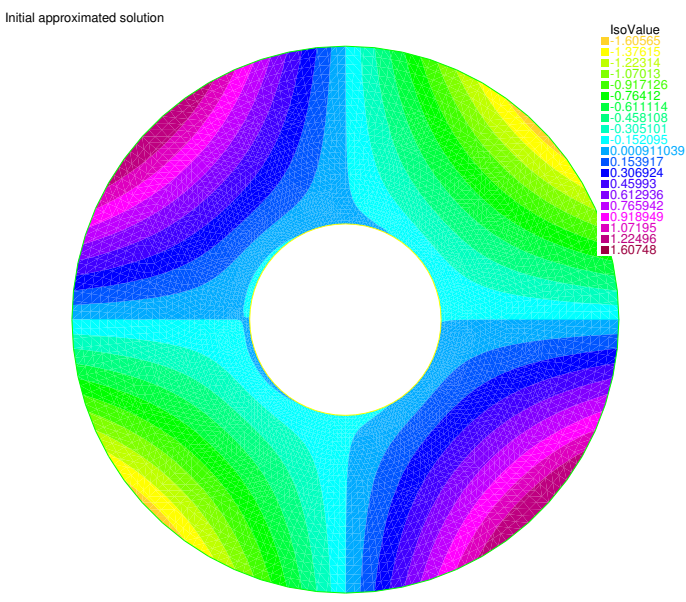

(b) Initial solution

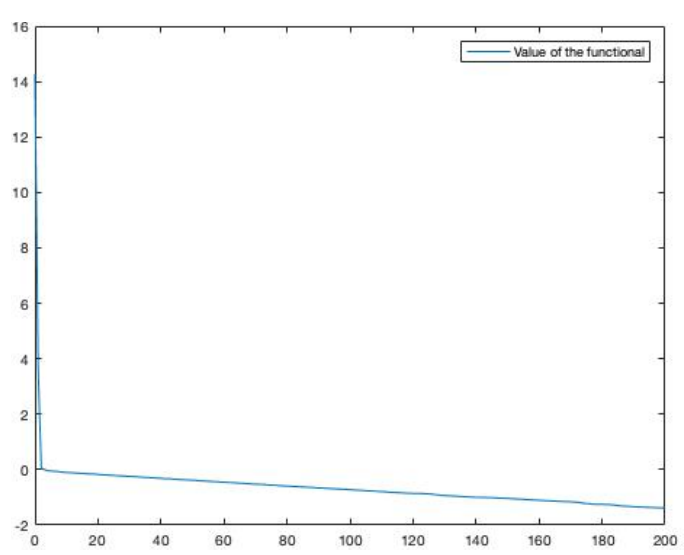

(d) Value of the functional

Figure 4: Simulations when $\Gamma_{\mathrm{c}}$ is the exterior boundary, with $1 \%$ of noise.

Now, from Theorem 1.5 , we known that

$$
\int_{\Omega}\left|\nabla v_{1}\left(\theta_{\mathrm{o}}\right)-\nabla v_{2}\left(\theta_{\mathrm{o}}\right)-\boldsymbol{F}^{\delta}\right|^{2} \mathrm{~d} x=\int_{\Omega}\left|\nabla v_{\varphi_{\mathrm{o}}}-\nabla v_{\psi_{\mathrm{o}}}+\nabla u_{\mathrm{D}}^{\delta}-\nabla u_{\mathrm{N}}^{\delta}\right|^{2} \mathrm{~d} x=c^{2} \delta^{2},
$$

where we recall that $u_{\mathrm{D}}^{\delta}$ and $u_{\mathrm{N}}^{\delta}$ are defined by 1.2 with $g_{\mathrm{D}}$ and $g_{\mathrm{N}}$ replaced by their noisy counterparts $g_{\mathrm{D}}^{\delta}$ and $g_{\mathrm{N}}^{\delta}$. It is not difficult to see from their respective definitions that actually $u_{\mathrm{o}}=v_{\varphi_{\mathrm{o}}}+u_{\mathrm{D}}^{\delta}$ and that $\tilde{u}=v_{\psi_{\mathrm{o}}}+u_{\mathrm{N}}^{\delta}$ is harmonic in $\Omega$ and verifies $\partial_{\nu} \tilde{u}_{\mid \Gamma}=g_{\mathrm{N}}^{\delta}$. Hence we have, using the continuity of the trace and the above equality,

$$
\left\|\partial_{\nu} u_{\mathrm{o}}-g_{\mathrm{N}}^{\delta}\right\|_{\mathrm{H}^{-1 / 2}(\Gamma)} \leqslant C\left\|\nabla\left(u_{\mathrm{o}}-\tilde{u}\right)\right\|_{\mathrm{L}^{2}(\Omega)} \leqslant C \delta,
$$

for some constant $C>0$.

Finally, $u_{\mathrm{o}}-u_{\mathrm{ex}}$ is a harmonic in $\Omega$, uniformly bounded for $\delta \in(0,1)$, and satisfies

$$
\left\|u_{\mathrm{o}}-u_{\mathrm{ex}}\right\|_{\mathrm{H}^{1 / 2}(\Gamma)}=\left\|g_{\mathrm{D}}^{\delta}-g_{\mathrm{D}}\right\|_{\mathrm{H}^{1 / 2}(\Gamma)} \leqslant \delta,
$$




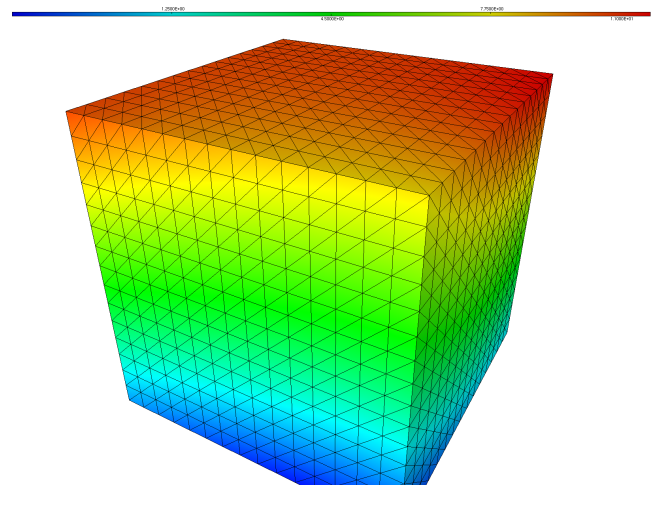

(a) Exact solution

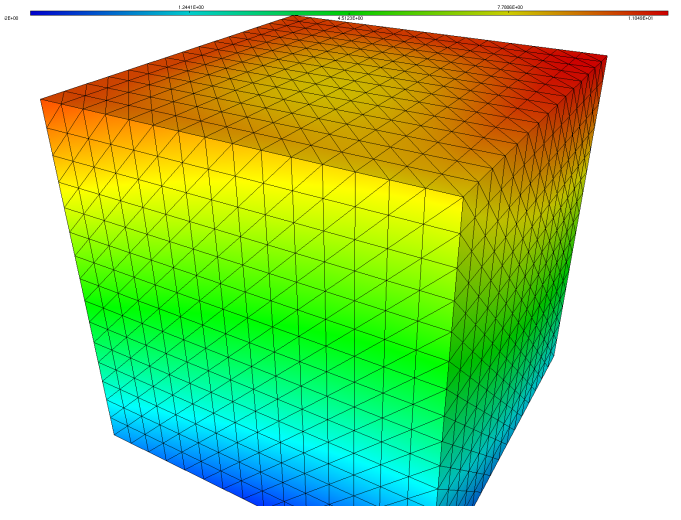

(c) Approximated solution

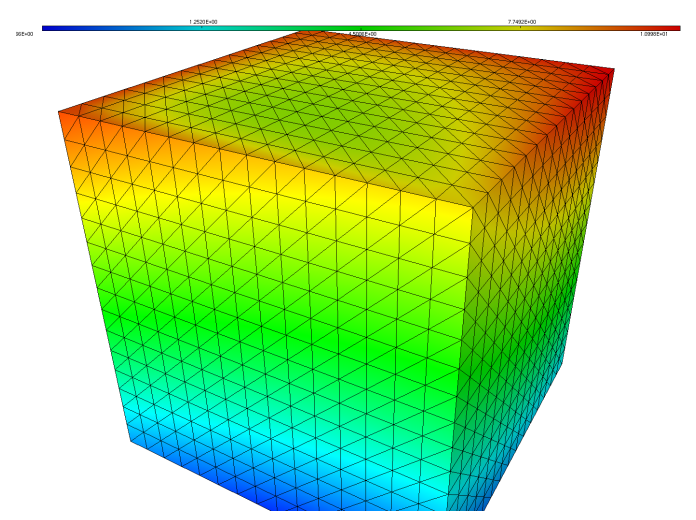

(b) Initial solution

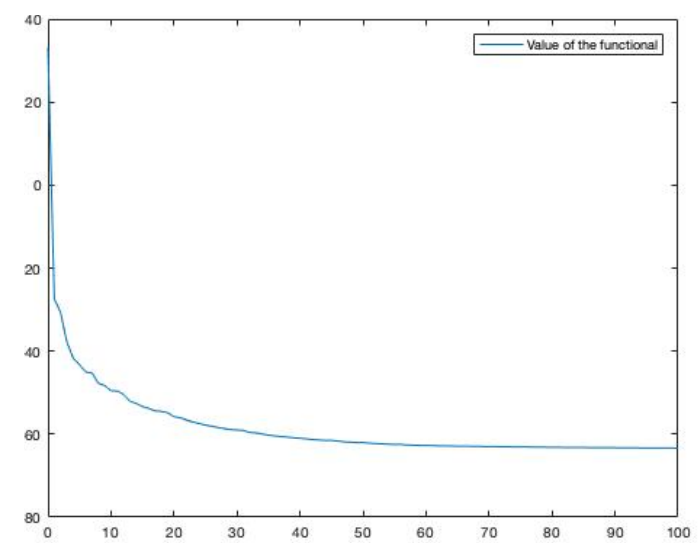

(d) Value of the functional

Figure 5: Simulations in the three dimensional case, with $1 \%$ of noise.

and

$$
\left\|\partial_{\nu}\left(u_{\mathrm{o}}-u_{\mathrm{ex}}\right)\right\|_{\mathrm{H}^{-1 / 2}(\Gamma)} \leqslant\left\|\partial_{\nu} u_{\mathrm{o}}-g_{\mathrm{N}}^{\delta}\right\|_{\mathrm{H}^{-1 / 2}(\Gamma)}+\left\|g_{\mathrm{N}}^{\delta}-g_{\mathrm{N}}\right\|_{\mathrm{H}^{-1 / 2}(\Gamma)} \leqslant C \delta .
$$

The result is then obtained by applying Theorem 1.1 with $u=u_{\mathrm{o}}-u_{\mathrm{ex}}$.

Remark 6.2. The logarithmic rate of convergence, very slow, is characteristic of the ill-posedness of Problem (1.1). Note also that even if $u_{\mathrm{o}}$ converges to $u_{\mathrm{ex}}$ strongly in $\mathrm{H}^{1}$, we only obtain a convergence rate in the weaker $\mathrm{L}^{2}$ norm.

\subsection{Imposing exactly a finite dimensional subpart of the data}

Even if the data at hand is noisy, some subpart of the data might be trustworthy. For example, if we decompose the data in some Fourier-type series, the low frequency part of the data is usually less affected by the noise than the high frequency part. In that situation, we might want to obtain a regularized solution of our inverse problem that corresponds exactly to the part of the data we trust. This is the topic of this section. 
In order to be more general, we present the results in the abstract setting of Section 3 let $y_{\mathrm{s}} \in \mathscr{Y}$ be the exact data and $x_{\mathrm{s}} \in \mathscr{A}$ the corresponding solution (i.e. $\mathscr{A} x_{\mathrm{s}}=y_{\mathrm{s}}$ ). We recall that $y^{\eta} \in \mathscr{Y}$ is the noisy data, verifying, for a given $\eta>0$,

$$
\left\|y^{\eta}-y_{\mathrm{s}}\right\| \mathscr{Y} \leqslant \eta
$$

Additionally, let $P: \mathscr{Y} \rightarrow \mathscr{Y}$ be an orthogonal projection, such that $\operatorname{rank}(P)<\infty$. We suppose that

$$
P y^{\eta}=P y_{\mathrm{s}} .
$$

In our setting, $P y^{\eta}$ is the trustworthy part of our data, which corresponds exactly to $P y_{\mathrm{s}}$, while $\left(\mathrm{I}_{d}-P\right) y^{\eta}$ is the part of the data which is really affected by the noise. We modify our Morozov set of regularized solutions:

$$
\mathscr{M}_{P}=\left\{x \in \mathscr{X},\left\|\mathscr{A} x-y^{\eta}\right\| \mathscr{Y} \leqslant \eta, P \mathscr{A} x=P y^{\eta}\right\} .
$$

In another word, we seek for an approximated solution that solves the problem up to the level of noise, but solves exactly the problem on the trustworthy part of the data. Note that $\mathscr{M}_{P}$ is not empty as $x_{\mathrm{s}} \in \mathscr{M}_{P}$.

It turns out that we only need a minor modification of our dual method to obtain exactly such a solution. It suffices to minimize the modified functional

$$
\mathscr{J}_{P}: y \in \mathscr{Y} \longmapsto \frac{1}{2}\left\|\mathscr{A}^{*} y\right\|_{\mathscr{X}}^{2}+\eta\left\|\left(\mathrm{I}_{d}-P\right) y\right\|_{\mathscr{Y}}-\left(y, y^{\eta}\right) \mathscr{Y}
$$

Let us begin by proving the well-posedness of this optimization problem.

Proposition 6.3. There exists a unique $y_{\mathrm{o}} \in \mathscr{Y}$ such that

$$
y_{\mathrm{o}}=\underset{y \in \mathscr{Y}}{\arg \min } \mathscr{J}_{P}(y) .
$$

Proof. The functional $\mathscr{J}_{P}$ being continuous and strictly convex (as $\mathscr{A}^{*}$ is one-to-one), we only need to prove that it is coercive. To do so, we follow the argument to absurdity of the proof of Proposition 3.2 We introduce the same sequence $\left(y_{n}\right)_{n \in \mathbb{N}}$ that verifies

$$
\lim _{n \rightarrow \infty}\left\|y_{n}\right\|_{\mathscr{Y}}=\infty \quad \text { and } \quad \mathscr{J}_{P}\left(y_{n}\right)<C,
$$

for a constant $C \in \mathbb{R}$, and then defined, for all $n \in \mathbb{N}, z_{n}=y_{n}\left\|y_{n}\right\|_{\mathscr{Y}}^{-1}$, which, as in the proof of Proposition 3.2 , weakly converges (up to a subsequence) to $0 \mathscr{Y}$.

Now the proof slightly changes. We first note that, as $\operatorname{rank}(P)<\infty$, up to a subsequence $P z_{n}$ strongly converges to $0_{\mathscr{Y}}$. Therefore, $\left(\mathrm{I}_{d}-P\right) z_{n}$ does not converges strongly to zero in $\mathscr{Y}$, otherwise a subsequence of $z_{n}$ would strongly converge to $0 \mathscr{Y}$, which is impossible as $\left\|z_{n}\right\|_{\mathscr{Y}}=1$.

Finally, as

$$
\mathscr{J}_{P}\left(y_{n}\right)>\left\|y_{n}\right\|_{\mathscr{Y}}\left[\eta\left\|\left(\mathrm{I}_{d}-P\right) z_{n}\right\|_{\mathscr{Y}}-\left(z_{n}, y^{\eta}\right)_{\mathscr{Y}}\right],
$$

the contradiction follows by letting $n$ goes to infinity.

Remark 6.4. The results remains true if we replace the projection $P$ by any compact operator $K$.

Let us now prove three propositions that will permit to obtain our main convergence theorem 6.8 To do so, let us introduce, as in Section 3 .

$$
x_{\mathrm{o}}=\mathscr{A}^{*} y_{\mathrm{o}}
$$


Proposition 6.5. The regularized solution $x_{\mathrm{o}}$ belongs to $\mathscr{M}_{P}$. Furthermore, if $P y_{\mathrm{o}} \neq y_{\mathrm{o}}$, then $x_{\mathrm{o}}$ verifies

$$
\mathscr{A} x_{\mathrm{o}}-y^{\eta}=\eta \frac{\left(P-\mathrm{I}_{d}\right) y_{\mathrm{o}}}{\left\|\left(P-\mathrm{I}_{d}\right) y_{\mathrm{o}}\right\|_{\mathscr{Y}}} .
$$

Proof. Suppose $P y_{\mathrm{o}} \neq y_{\mathrm{o}}$. Then $\mathscr{J}_{P}$ is differentiable at $y_{\mathrm{o}}$, and the Euler-Lagrange equation associated to our minimization problem gives

$$
\mathscr{A} \mathscr{A}^{*} y_{\mathrm{o}}+\eta \frac{\left(\mathrm{I}_{d}-P\right) y_{\mathrm{o}}}{\left\|\left(\mathrm{I}_{d}-P\right) y_{\mathrm{o}}\right\| \mathscr{Y}}-y^{\eta}=0 \mathscr{Y}
$$

The results follows since we can deduce from this equality that, additionally, $x_{\mathrm{o}} \in \mathscr{M}_{P}$.

The case $P y_{\mathrm{o}}=y_{\mathrm{o}}$ is slightly more delicate. First of all, even if $\mathscr{J}_{P}$ is not anymore differentiable at $y_{0}$, we recall that, since $\mathscr{J}_{P}\left(y_{\mathrm{o}}\right)=\min _{y \in \mathscr{Y}} \mathscr{J}_{P}(y), 0_{\mathscr{Y}}$ belongs to the sub-differential of $\mathscr{J}_{P}$ at $y_{\mathrm{o}}$ (see, e.g., [29, Section 5 p.20]), or equivalently

$$
\mathscr{A} \mathscr{A}^{*} y_{\mathrm{o}}-y^{\eta} \in \eta \overline{\mathcal{B}}_{1},
$$

where $\overline{\mathcal{B}}_{1}$ is the closed unit ball of $\mathscr{Y}$. Hence $\left\|\mathscr{A} x_{\mathrm{o}}-y^{\eta}\right\| \mathscr{Y} \leqslant \eta$. Furthermore, we note that

$$
\mathscr{J}_{P}\left(y_{\mathrm{o}}\right)=\min _{y \in \mathscr{Y}} \mathscr{J}_{P}(y) \leqslant \min _{y \in \operatorname{Im}(P)} \mathscr{J}_{P}(y)=\min _{y \in \operatorname{Im}(P)} \frac{1}{2}\left\|\mathscr{A}^{*} y\right\|_{\mathscr{X}}^{2}-\left(y^{\eta}, y\right) \mathscr{Y},
$$

which easily implies, since $y_{\mathrm{o}} \in \operatorname{Im}(P)$,

$$
y_{\mathrm{o}}=\underset{y \in \operatorname{Im}(P)}{\arg \min } \frac{1}{2}\left\|\mathscr{A}^{*} y\right\|_{\mathscr{X}}^{2}-\left(y^{\eta}, y\right) \mathscr{Y} .
$$

The Euler-Lagrange equation associated with this minimization problem leads to

$$
\left(\mathscr{A}^{*} y_{\mathrm{o}}, \mathscr{A}^{*} y\right)_{\mathscr{X}}-\left(y^{\eta}, y\right)_{\mathscr{Y}}=0=\left(\mathscr{A} x_{\mathrm{o}}-y^{\eta}, y\right)_{\mathscr{Y}}, \quad \forall y \in \operatorname{Im}(P) .
$$

Hence $\mathscr{A} x_{\mathrm{o}}-y^{\eta}$ belongs to the kernel of $P$, which proves that $x_{\mathrm{o}} \in \mathscr{M}_{P}$.

Proposition 6.6. We have

$$
\left\|x_{\mathrm{o}}\right\|_{\mathscr{X}}^{2}=-2 \mathscr{J}_{P}\left(y_{\mathrm{o}}\right)
$$

Proof. In the case $P y_{\mathrm{o}} \neq y_{\mathrm{o}}$, the proof is precisely the one of Proposition 3.5. In the other case, we have

$$
\mathscr{J}_{P}\left(y_{\mathrm{o}}\right)=\frac{1}{2}\left\|x_{\mathrm{o}}\right\|_{\mathscr{X}}^{2}-\left(y^{\eta}, y_{\mathrm{o}}\right) \mathscr{Y}
$$

and as shown in the previous proof of Proposition 6.5.

$$
y_{\mathrm{o}}=\underset{y \in \operatorname{Im}(P)}{\arg \min } \frac{1}{2}\left\|\mathscr{A}^{*} y\right\|_{\mathscr{X}}^{2}-\left(y^{\eta}, y\right) \mathscr{Y} .
$$

Then, using the Euler-Lagrange equation associated to this minimization problem (see (6.1)), we deduce that

$$
\left\|x_{\mathrm{o}}\right\|_{\mathscr{X}}^{2}=\left\|\mathscr{A}^{*} y_{\mathrm{o}}\right\|_{\mathscr{X}}^{2}=\left(y^{\eta}, y_{\mathrm{o}}\right)_{\mathscr{Y}},
$$

and the result follows.

Proposition 6.7. Let $x \in \mathscr{M}_{P}, x \neq x_{\mathrm{o}}$. Then $\|x\|_{\mathscr{X}}>\left\|x_{\mathrm{o}}\right\|_{\mathscr{X}}$. 
Proof. The proof is almost exactly the same as the one of Proposition 3.6. Let $x \in \mathscr{M}_{P}$, with $x \neq x_{\mathrm{o}}$, and define $y_{p}=y^{\eta}-\mathscr{A} x$, which by definition verifies

$$
P y_{p}=0 \mathscr{Y} \quad \text { and } \quad\left\|y_{p}\right\|_{\mathscr{Y}} \leqslant \eta \text {. }
$$

Then, using Proposition 6.6 .

$$
\begin{aligned}
& \frac{1}{2}\left(\|x\|_{\mathscr{X}}^{2}-\left\|x_{\mathrm{o}}\right\|_{\mathscr{X}}^{2}\right)= \frac{1}{2}\|x\|_{\mathscr{X}}^{2}+\mathscr{J}\left(y_{\mathrm{o}}\right)=\frac{1}{2}\|x\|_{\mathscr{X}}^{2}+\frac{1}{2}\left\|\mathscr{A}^{*} y_{\mathrm{o}}\right\|_{\mathscr{X}}^{2}+\eta\left\|\left(\mathrm{I}_{d}-P\right) y_{\mathrm{o}}\right\|_{\mathscr{Y}}-\left(y_{\mathrm{o}}, y^{\eta}\right)_{\mathscr{Y}} \\
&= \frac{1}{2}\|x\|_{\mathscr{X}}^{2}+\frac{1}{2}\left\|x_{\mathrm{o}}\right\|_{\mathscr{X}}^{2}+\eta\left\|\left(\mathrm{I}_{d}-P\right) y_{\mathrm{o}}\right\|_{\mathscr{Y}}-\left(y_{\mathrm{o}}, \mathscr{A} x+y_{p}\right)_{\mathscr{Y}} \\
&=\frac{1}{2}\|x\|_{\mathscr{X}}^{2}+\frac{1}{2}\left\|x_{\mathrm{o}}\right\|_{\mathscr{X}}^{2}-\left(\mathscr{A}^{*} y_{\mathrm{o}}, x\right) \mathscr{X}+\eta\left\|\left(\mathrm{I}_{d}-P\right) y_{\mathrm{o}}\right\| \mathscr{Y}-\left(y_{\mathrm{o}},\left(\mathrm{I}_{d}-P\right) y_{p}\right) \mathscr{Y} \\
&=\underbrace{\frac{1}{2}\|x\|_{\mathscr{X}}^{2}+\frac{1}{2}\left\|x_{\mathrm{o}}\right\|_{\mathscr{X}}^{2}-\left(x_{\mathrm{o}}, x\right) \mathscr{X}}_{=\frac{1}{2}\left\|x-x_{\mathrm{o}}\right\|_{\mathscr{X}}^{2}>0}+\underbrace{\eta\left\|\left(\mathrm{I}_{d}-P\right) y_{\mathrm{o}}\right\| \mathscr{Y}-\left(\left(\mathrm{I}_{d}-P\right) y_{\mathrm{o}}, y_{p}\right) \mathscr{Y}}_{\geqslant 0},
\end{aligned}
$$

which ends the proof.

We can now state our convergence theorem, which can be proven exactly as Theorem 3.8 thanks to the previous propositions.

Theorem 6.8. The regularized solution $x_{\mathrm{o}}$ converges to $x_{\mathrm{s}}$ as $\eta$ goes to zero.

As a conclusion, if $P y^{\eta}=P y_{\mathrm{s}}$, then minimizing the modified functional $\mathscr{J}_{P}$ leads to a regularized solution that satisfies both the constraints

$$
\left\|\mathscr{A} x_{\mathrm{o}}-y^{\eta}\right\|_{\mathscr{Y}} \leqslant \eta, \quad \text { and } \quad P \mathscr{A} x_{\mathrm{o}}=P y^{\eta}=P y_{\mathrm{s}},
$$

without numerical difficulties since the main minimization problem remains without constraint.

\section{A Functional framework}

In this appendix, we precise the functional framework used in the present study, in particular the functional spaces defined on open subparts of the boundary of $\Omega$.

\section{A.1 Functional spaces on the boundary}

Let $\Sigma$ be an open subset of $\partial \Omega$ of positive Lebesgue measure. As usual, we denote by $\mathrm{H}^{1 / 2}(\Sigma)$ the set of functions of $\mathrm{L}^{2}(\Sigma)$ which are the trace on $\Sigma$ of functions of $\mathrm{H}^{1}(\Omega)$ :

$$
\mathrm{H}^{1 / 2}(\Sigma)=\left\{g \in \mathrm{L}^{2}(\Sigma), \exists w \in \mathrm{H}^{1}(\Omega), w_{\mid \Sigma}=g\right\} .
$$

The space $\mathrm{H}^{1 / 2}(\Sigma)$ endowed with the usual norm,

$$
\|g\|_{\mathrm{H}^{1 / 2}(\Sigma)}=\inf _{w \in \mathrm{H}^{1}(\Omega), w_{\mid \Sigma}=g}\|w\|_{\mathrm{H}^{1}(\Omega)},
$$

is a Banach space. We note

$$
\mathrm{H}_{\diamond}^{1 / 2}(\Sigma)=\left\{g \in \mathrm{H}^{1 / 2}(\Sigma), \quad \int_{\Sigma} g \mathrm{~d} s=0\right\}
$$


which is a closed subspace of $\mathrm{H}^{1 / 2}(\Sigma)$. Note that thanks to Poincaré inequality, there exists a constant $C>0$ such that for all $g$ in $\mathrm{H}_{\diamond}^{1 / 2}(\Sigma)$, all $v \in \mathrm{H}^{1}(\Omega)$ such that $v_{\mid \Sigma}=g$, one has

$$
\|g\|_{\mathrm{H}^{1 / 2}(\Sigma)} \leqslant C\|v\|_{\mathrm{H}^{1}(\Omega)} \leqslant C\|\nabla v\|_{\mathbf{L}^{2}(\Omega)} .
$$

We also define

$$
\tilde{\mathrm{H}}^{1 / 2}(\Sigma)=\mathrm{H}^{1 / 2}(\Sigma) / \mathbb{R}
$$

which, endowed with the norm

$$
\|g\|_{\tilde{\mathrm{H}}^{1 / 2}(\Sigma)}=\inf _{c \in \mathbb{R}}\|g-c\|_{\mathrm{H}^{1 / 2}(\Sigma)},
$$

is also a Banach space. Clearly, we have, for all $\left(g_{1}, g_{2}\right) \in \mathrm{H}^{1 / 2}(\Sigma)^{2}$,

$$
g_{1}=g_{2} \in \tilde{\mathrm{H}}^{1 / 2}(\Sigma) \Longleftrightarrow g_{1}=g_{2}+c \text { for some real constant } c .
$$

Following [37, we define

$$
\mathrm{H}_{00}^{1 / 2}(\Sigma)=\left\{g \in \mathrm{L}^{2}(\Sigma), g_{\text {ext }} \in \mathrm{H}^{1 / 2}(\partial \Omega)\right\} \subset \mathrm{H}^{1 / 2}(\Sigma),
$$

where $g_{\text {ext }} \in \mathrm{L}^{2}(\partial \Omega)$ is defined as

$$
g_{\mathrm{ext}}=g \text { on } \Sigma, \quad \text { and } \quad g_{\mathrm{ext}}=0 \text { on } \partial \Omega \backslash \bar{\Sigma},
$$

and $\mathrm{H}^{-1 / 2}(\Sigma)$ as the dual space of $\mathrm{H}_{00}^{1 / 2}(\Sigma)$, endowed with the dual norm

$$
\|h\|_{\mathrm{H}^{-1 / 2}(\Sigma)}=\sup _{g \in \mathrm{H}_{00}^{1 / 2}(\Sigma),\left\|g_{\mathrm{ext}}\right\|_{\mathrm{H}^{1 / 2}(\partial \Omega)}=1}\langle h, g\rangle,
$$

the bracket meaning the dual evaluation between $\mathrm{H}^{-1 / 2}(\Sigma)$ and $\mathrm{H}_{00}^{1 / 2}(\Sigma)$. Note that by construction, one has

$$
\mathrm{H}_{\diamond}^{1 / 2}(\Sigma) \subset \mathrm{H}^{1 / 2}(\Sigma) \subset \mathrm{L}^{2}(\Sigma) \subset \mathrm{H}^{-1 / 2}(\Sigma) .
$$

Furthermore, thanks to Green formula, we know that for all $v \in \mathrm{H}^{1}(\Omega)$ such that $\Delta v \in \mathrm{L}^{2}(\Omega), \partial_{\nu} v_{\mid \Sigma}$ belongs to $\mathrm{H}^{-1 / 2}(\Sigma)$ and

$$
\left\|\partial_{\nu} v_{\mid \Sigma}\right\|_{\mathrm{H}^{-1 / 2}(\Sigma)} \leqslant c\left(\|v\|_{\mathrm{H}^{1}(\Omega)}+\|\Delta v\|_{\mathrm{L}^{2}(\Omega)}\right) .
$$

We now suppose that $\partial \Omega=\Sigma \cup \Sigma_{\mathrm{c}}$, with $\Sigma$ and $\Sigma_{\mathrm{c}}$ be two open subsets and of positive Lebesgue measure, and $\Sigma \cap \Sigma_{\mathrm{c}}=\varnothing$. For $g \in \mathrm{H}_{\diamond}^{1 / 2}(\Sigma)$ and $h \in \mathrm{H}^{-1 / 2}\left(\Sigma_{\mathrm{c}}\right)$, we define the following problem: find $u$ in $\mathrm{H}^{1}(\Omega)$ such that

$$
\left(\mathscr{P}_{u}\right)\left\{\begin{aligned}
\Delta u & =0 \text { in } \Omega, \\
u & =g \text { on } \Sigma, \\
\partial_{\nu} u & =h \text { on } \Sigma_{\mathrm{c}} .
\end{aligned}\right.
$$

Lemma A.1. There exists a unique $u \in \mathrm{H}^{1}(\Omega)$ solution of $\mathscr{P}_{u}$. Furthermore, there exists a positive constant $c$ such that

$$
\|u\|_{\mathrm{H}^{1}(\Omega)} \leqslant c\left(\|g\|_{\mathrm{H}^{1 / 2}(\Sigma)}+\|h\|_{\mathrm{H}^{-1 / 2}\left(\Gamma_{\mathrm{c}}\right)}\right) .
$$

Proof. It is not difficult to prove that there exists a unique $R(g) \in \mathrm{H}^{1}(\Omega)$ satisfying $R(g)_{\mid \Sigma}=g$ and $\|R(g)\|_{\mathrm{H}^{1}(\Omega)}=\|g\|_{\mathrm{H}^{1 / 2}(\Sigma)}$. We then denote

$$
\mathrm{H}_{\Sigma}^{1}(\Omega)=\left\{v \in \mathrm{H}^{1}(\Omega), v_{\mid \Sigma}=0\right\}
$$


which is a closed subspace of $\mathrm{H}^{1}(\Omega)$, hence an Hilbert space when endowed by the $\mathrm{H}^{1}$-scalar product. But thanks to Poincaré inequality, the $\mathrm{H}^{1}$-semi-norm is an equivalent norm on $\mathrm{H}_{\Sigma}^{1}(\Omega)$.

By definition, for all $v \in \mathrm{H}_{\Sigma}^{1}(\Omega), v_{\mid \Sigma_{\mathrm{c}}}$ belongs to $\mathrm{H}_{00}^{1 / 2}\left(\Sigma_{\mathrm{c}}\right)$, hence $\left\langle h, v_{\mid \Sigma_{\mathrm{c}}}\right\rangle$ is well defined. From Lax-Milgram theorem, there exists a unique $w \in \mathrm{H}_{\Sigma}^{1}(\Omega)$ such that for all $v \in \mathrm{H}_{\Sigma}^{1}(\Omega)$,

$$
\int_{\Omega} \nabla w \cdot \nabla v \mathrm{~d} x=-\int_{\Omega} \nabla R(g) \cdot \nabla v \mathrm{~d} x+\left\langle h, v_{\mid \Sigma_{\mathrm{c}}}\right\rangle,
$$

which furthermore verifies

$$
\|w\|_{\mathrm{H}^{1}(\Omega)} \leqslant c\left(\|g\|_{\mathrm{H}^{1 / 2}(\Sigma)}+\|h\|_{\mathrm{H}^{-1 / 2}\left(\Sigma_{\mathrm{c}}\right)}\right) .
$$

In particular, by linearity of Problem $\left(\mathscr{P}_{u}\right)$, we have obtained the uniqueness property of Lemma A.1.

We note that $u=w+R(g)$ satisfies by construction $\Delta u=0, u_{\mid \Sigma}=g$ and

$$
\|u\|_{\mathrm{H}^{1}(\Omega)} \leqslant c\left(\|g\|_{\mathrm{H}^{1 / 2}(\Sigma)}+\|h\|_{\mathrm{H}^{-1 / 2}\left(\Sigma_{\mathrm{c}}\right)}\right) .
$$

Furthermore, using Green formula and the variational problem satisfied by $w$, we obtain that for all $\tilde{g} \in \mathrm{H}_{00}^{1 / 2}\left(\Sigma_{\mathrm{c}}\right)$,

$$
\left\langle\partial_{\nu} u, \tilde{g}_{\text {ext }}\right\rangle=\langle h, \tilde{g}\rangle,
$$

hence $\partial_{\nu} u_{\mid \Sigma_{\mathrm{c}}}=h$, which ends the proof.

For $\psi$ in $\mathrm{H}_{\diamond}^{1 / 2}(\Sigma)$ and $\varphi \in \mathrm{H}^{-1 / 2}\left(\Sigma_{\mathrm{c}}\right)$, we define $v_{\psi}$ the unique solution of $\left(\mathscr{P}_{u}\right)$ with $g=\psi$ and $h=0$, and symmetrically, we denote $v_{\varphi}$ the unique solution of $\left(\mathscr{P}_{u}\right)$ with $g=0$ and $h=\varphi$.

Lemma A.2. The application

$$
\{\cdot, \cdot\}:\left(\psi_{1}, \psi_{2}\right) \in \mathrm{H}_{\diamond}^{1 / 2}(\Sigma) \times \mathrm{H}_{\diamond}^{1 / 2}(\Sigma) \longmapsto\left\{\psi_{1}, \psi_{2}\right\}=\int_{\Omega} \nabla v_{\psi_{1}} \cdot \nabla v_{\psi_{2}} \mathrm{~d} x
$$

defines a scalar product on $\mathrm{H}_{\diamond}^{1 / 2}(\Sigma)$, the corresponding norm being equivalent to the standard norm. Therefore, $\left(\mathrm{H}_{\diamond}^{1 / 2}(\Sigma),\{\cdot, \cdot\}\right)$ is a Hilbert space.

Proof. It is not difficult to see that $\{\cdot, \cdot\}$ is bilinear symmetric positive. It is definite as if $\{\psi, \psi\}=0$, then $\nabla v_{\psi}=0$, hence $v_{\psi}=\alpha \in \mathbb{R}$. But as $\psi=v_{\psi \mid \Sigma}=\alpha$ is mean free, this immediately implies $\alpha=0$.

Now, on one side, from the continuity of trace, we get $\|\psi\|_{\mathrm{H}^{1 / 2}(\Sigma)} \leqslant c\left\|v_{\psi}\right\|_{\mathrm{H}^{1}(\Omega)}$. But as $v_{\psi \mid \Sigma}=\psi$ is mean free, from a Poincaré-type inequality we obtain $\left\|v_{\psi}\right\|_{\mathrm{H}^{1}(\Omega)} \leqslant c\left\|\nabla v_{\psi}\right\|_{\mathrm{L}^{2}(\Omega)}$. So, using finally Lemma A.1. we obtain two positive constants $c_{1}$ and $c_{2}$ so that

$$
c_{1}\|\psi\|_{\mathrm{H}^{1 / 2}(\Sigma)} \leqslant\left\|\nabla v_{\psi}\right\|_{\mathrm{H}^{1}(\Omega)} \leqslant c_{2}\|\psi\|_{\mathrm{H}^{1 / 2}(\Sigma)},
$$

which ends the proof.

Lemma A.3. The application

$$
\{\cdot, \cdot\}:\left(\varphi_{1}, \varphi_{2}\right) \in \mathrm{H}^{-1 / 2}\left(\Sigma_{\mathrm{c}}\right) \times \mathrm{H}^{-1 / 2}\left(\Sigma_{\mathrm{c}}\right) \longmapsto\left\{\varphi_{1}, \varphi_{2}\right\}=\int_{\Omega} \nabla v_{\varphi_{1}} \cdot \nabla v_{\varphi_{2}} \mathrm{~d} x,
$$

defines a scalar product on $\mathrm{H}^{-1 / 2}\left(\Sigma_{\mathrm{c}}\right)$, the corresponding norm being equivalent to the standard norm. Therefore, $\left(\mathrm{H}^{-1 / 2}\left(\Sigma_{\mathrm{c}}\right),\{\cdot, \cdot\}\right)$ is a Hilbert space. 
Proof. It is not difficult to prove that $\{\cdot, \cdot\}$ is indeed a scalar product on $\mathrm{H}^{-1 / 2}\left(\Sigma_{\mathrm{c}}\right)$, using that by definition, $v_{\varphi \mid \Sigma}=0$.

To prove the equivalence of the norms, we first note that by continuity of the normal derivative, the fact that by definition $v_{\varphi}$ is harmonic in $\Omega$, and a Poincaré-like inequality as $v_{\varphi \mid \Sigma}=0$, we obtain

$$
\|\varphi\|_{\mathrm{H}^{-1 / 2}\left(\Sigma_{\mathrm{c}}\right)} \leqslant c\left(\left\|v_{\varphi}\right\|_{\mathrm{H}^{1}(\Omega)}+\left\|\Delta v_{\varphi}\right\|_{\mathrm{L}^{2}(\Omega)}\right)=c\left\|v_{\varphi}\right\|_{\mathrm{H}^{1}(\Omega)} \leqslant c\left\|\nabla v_{\varphi}\right\|_{\mathrm{L}^{2}(\Omega)} .
$$

On the other hand, Lemma A.1 gives

$$
\left\|\nabla v_{\varphi}\right\|_{\mathrm{L}^{2}(\Omega)} \leqslant c\|\varphi\|_{\mathrm{H}^{-1 / 2}\left(\Sigma_{\mathrm{c}}\right)} .
$$

The result follows.

\section{A.2 Functional space in the volume}

We define

$$
\mathbf{H}(\Omega)=\left\{\nabla w, w \in \mathrm{H}^{1}(\Omega) \text { satisfies } \Delta w=0 \text { in } \Omega\right\} \subset \mathbf{L}^{2}(\Omega) .
$$

Lemma A.4. The space $\mathbf{H}(\Omega)$, endowed with the usual scalar product of $\mathbf{L}^{2}(\Omega)$, is a Hilbert space.

Proof. As $\mathbf{H}(\Omega)$ is a subspace of $\mathbf{L}^{2}(\Omega)$, it is sufficient to prove that it is closed for the $\mathbf{L}^{2}$-norm. Therefore, let $\boldsymbol{p}_{n}$ a sequence of elements of $\mathbf{H}(\Omega)$ converging to some $\boldsymbol{p} \in \mathbf{L}^{2}(\Omega)$ :

$$
\lim _{n \rightarrow \infty}\left\|\boldsymbol{p}_{n}-\boldsymbol{p}\right\|_{\mathbf{L}^{2}(\Omega)}=0 .
$$

By definition, there exists a sequence of harmonic functions $v_{n} \in \mathrm{H}^{1}(\Omega)$ such that $\boldsymbol{p}_{n}=\nabla v_{n}$. The sequence

$$
\tilde{v}_{n}=v_{n}-\frac{1}{|\Omega|} \int_{\Omega} v_{n} \mathrm{~d} x
$$

is also a sequence of harmonic functions such that $\nabla \tilde{v}_{n}=\boldsymbol{p}_{n}$. From Poincaré-Wirtinger inequality and the fact that $\boldsymbol{p}_{n}$ converges to $\boldsymbol{p}$, we deduce that $\tilde{v}_{n}$ is a bounded sequence in $\mathrm{H}^{1}(\Omega)$, and therefore weakly converge to some $v \in \mathrm{H}^{1}(\Omega)$. As $\tilde{v}_{n}$ is harmonic for all $n$, so is $v$, hence $\nabla v$ is an element of $\mathbf{H}(\Omega)$. Finally, in $\mathbf{L}^{2}(\Omega), \nabla \tilde{v}_{n}$ weakly converges to $\nabla v$, and strongly converges to $\boldsymbol{p}$, hence $\boldsymbol{p}=\nabla v$, which ends the proof.

\section{References}

[1] R. Aboulä̈ch, A. Ben Abda, and M. Kallel, Missing boundary data reconstruction via an approximate optimal control, Inverse Probl. Imaging, 2 (2008), https://doi.org/10.3934/ipi. 2008.2 .411 .

[2] L. Afraites, M. Dambrine, K. Eppler, and D. Kateb, Detecting perfectly insulated obstacles by shape optimization techniques of order two, Discrete Contin. Dyn. Syst. Ser. B, 8 (2007), https://doi.org/10.3934/dcdsb.2007.8.389

[3] G. Alessandrini, L. Rondi, E. Rosset, and S. Vessella, The stability for the Cauchy problem for elliptic equations, Inverse Problems, 25 (2009), https://doi.org/10.1088/ 0266-5611/25/12/123004, https://arxiv.org/abs/0907.2882.

[4] S. Andrieux and T. N. Baranger, An energy error-based method for the resolution of the Cauchy problem in 3D linear elasticity, Comput. Methods Appl. Mech. Engrg., 197 (2008), https : //doi.org/10.1016/j.cma.2007.08.022 
[5] S. Andrieux, T. N. Baranger, And A. Ben Abda, Solving Cauchy problems by minimizing an energy-like functional, Inverse Problems, 22 (2006), https://doi.org/10.1088/0266-5611/ 22/1/007, https://hal. archives-ouvertes.fr/hal-00139569.

[6] M. Arioli and D. Loghin, Discrete interpolation norms with applications, SIAM J. Numer. Anal., 47 (2009), https://doi.org/10.1137/080729360

[7] M. Azaïez, F. Ben Belgacem, and H. El Fekih, On Cauchy's problem. II. Completion, regularization and approximation, Inverse Problems, 22 (2006), https://doi.org/10.1088/ 0266-5611/22/4/012

[8] F. Ben Belgacem, Why is the Cauchy problem severely ill-posed?, Inverse Problems, 23 (2007), https://doi.org/10.1088/0266-5611/23/2/020

[9] F. Ben Belgacem and H. El Fekih, On Cauchy's problem. I. A variational Steklov-Poincaré theory, Inverse Problems, 21 (2005), https://doi.org/10.1088/0266-5611/21/6/008.

[10] F. Ben Belgacem, H. El Fekih, and F. Jelassi, The Lavrentiev regularization of the data completion problem, Inverse Problems, 24 (2008), https://doi.org/10.1088/0266-5611/24/ $4 / 045009$.

[11] F. Ben Belgacem, V. Girault, and F. Jelassi, Analysis of Lavrentiev-finite element methods for data completion problems, Numer. Math., 139 (2018), https://doi.org/10.1007/ s00211-017-0930-6.

[12] Y. BOUKARI AND H. HADDAR, A convergent data completion algorithm using surface integral equations, Inverse Problems, 31 (2015), https://doi.org/10.1088/0266-5611/31/3/035011, https://hal.archives-ouvertes.fr/hal-01110005.

[13] L. Bourgeois And L. Chesnel, On quasi-reversibility solutions to the cauchy problem for the laplace equation: regularity and error estimates, June 2019, https://arxiv.org/abs/1906. 08700

[14] L. Bourgeois And J. DARdÉ, A duality-based method of quasi-reversibility to solve the Cauchy problem in the presence of noisy data, Inverse Problems, 26 (2010), https://doi.org/10.1088/ 0266-5611/26/9/095016.

[15] E. Burman, Stabilized finite element methods for nonsymmetric, noncoercive, and ill-posed problems. Part I: Elliptic equations, SIAM J. Sci. Comput., 35 (2013), https://doi.org/10. 1137/130916862, https://arxiv.org/abs/1304.2414.

[16] E. Burman, Error estimates for stabilized finite element methods applied to ill-posed problems, C. R. Math. Acad. Sci. Paris, 352 (2014), https://doi.org/10.1016/j.crma.2014.06.008 https://arxiv.org/abs/1406.4371.

[17] E. Burman, M. G. Larson, and L. Oksanen, Primal-dual mixed finite element methods for the elliptic Cauchy problem, SIAM J. Numer. Anal., 56 (2018), https://doi.org/10.1137/ 17M1163335, https://arxiv.org/abs/1712.10172.

[18] H. Cao, M. V. Klibanov, and S. V. Pereverzev, A Carleman estimate and the balancing principle in the quasi-reversibility method for solving the Cauchy problem for the Laplace equation, Inverse Problems, 25 (2009), https://doi.org/10.1088/0266-5611/25/3/035005. 
[19] F. Caubet, C. Conca, and M. Godoy, On the detection of several obstacles in 2D Stokes flow: topological sensitivity and combination with shape derivatives, Inverse Probl. Imaging, 10 (2016), https://doi.org/10.3934/ipi.2016003, https://hal.archives-ouvertes. fr/hal-01191099.

[20] F. Caubet, M. Dambrine, D. Kateb, and C. Z. Timimoun, A Kohn-Vogelius formulation to detect an obstacle immersed in a fluid, Inverse Probl. Imaging, 7 (2013), https://doi.org/ 10.3934/ipi.2013.7.123, https://hal.archives-ouvertes.fr/hal-00678036.

[21] F. Caubet, J. Dardé, and M. Gogoy, On the data completion problem and the inverse obstacle problem with partial cauchy data for laplace's equation, ESAIM: Control, Optimisation and Calculus of Variations, 25 (2019), https://doi.org/10.1051/cocv/2017056, https://hal. archives-ouvertes.fr/hal-01624524.

[22] S. Chanbane, M. Masmoudi, and H. Meftahi, Topological and shape gradient strategy for solving geometrical inverse problems, J. Math. Anal. Appl., 400 (2013), https://doi.org/10. $1016 / j \cdot j$ maa.2012.11.044

[23] R. Chapko and B. T. Johansson, Boundary-integral approach for the numerical solution of the Cauchy problem for the Laplace equation, Ukraïn. Mat. Zh., 68 (2016), https://doi.org/ 10.1007/s11253-017-1339-1. Reprinted in Ukrainian Math. J. 68 (2017), no. 12, 1929-1948.

[24] A. Cimetière, F. Delvare, M. Jaoua, and F. Pons, Solution of the Cauchy problem using iterated Tikhonov regularization, Inverse Problems, 17 (2001), https://doi.org/10.1088/ $0266-5611 / 17 / 3 / 313$

[25] J. DARDÉ, Iterated quasi-reversibility method applied to elliptic and parabolic data completion problems, Inverse Probl. Imaging, 10 (2016), https://doi.org/10.3934/ipi.2016005, https: //hal.archives-ouvertes.fr/hal-01136395.

[26] J. Dardé, A. Hannukainen, and N. Hyvönen, An $H_{\text {div }}$-based mixed quasi-reversibility method for solving elliptic Cauchy problems, SIAM J. Numer. Anal., 51 (2013), https://doi. org/10.1137/120895123.

[27] F. Delvare and A. Cimetière, Unique discrete harmonic continuation and data completion problems using the fading regularization method, Numer. Algorithms, 75 (2017), https://doi. org/10.1007/s11075-016-0218-4.

[28] P. Demeestère, A remark on the relation between the Tykhonov regularization and constraint relaxation for an optimal control problem, Appl. Math. Lett., 11 (1998), https://doi.org/10. 1016/S0893-9659(98)00085-8

[29] I. Ekeland and R. TÉmam, Convex analysis and variational problems, vol. 28 of Classics in Applied Mathematics, Society for Industrial and Applied Mathematics (SIAM), Philadelphia, PA, english ed., 1999, https://doi.org/10.1137/1.9781611971088. Translated from the French.

[30] H. W. Engl, M. Hanke, And A. Neubauer, Regularization of inverse problems, vol. 375 of Mathematics and its Applications, Kluwer Academic Publishers Group, Dordrecht, 1996.

[31] S. ERvedozA, Control issues and linear projection constraints on the control and on the controlled trajectory, Sept. 2019, https://arxiv.org/abs//1909.01649.

[32] J. HAdAmard, Lectures on Cauchy's problem in linear partial differential equations, Dover Publications, New York, 1953. 
[33] F. Hecht, New development in Freefem++, J. Numer. Math., 20 (2012), pp. 251-265.

[34] M. V. Klibanov, Carleman estimates for the regularization of ill-posed Cauchy problems, Appl. Numer. Math., 94 (2015), https://doi.org/10.1016/j.apnum.2015.02.003, https://arxiv. org/abs/1410.7521.

[35] M. V. Klibanov And F. SAntosa, A computational quasi-reversibility method for Cauchy problems for Laplace's equation, SIAM J. Appl. Math., 51 (1991), https://doi.org/10.1137/ 0151085 .

[36] R. Lattès And J.-L. Lions, The method of quasi-reversibility. Applications to partial differential equations, Translated from the French edition and edited by Richard Bellman. Modern Analytic and Computational Methods in Science and Mathematics, No. 18, American Elsevier Publishing Co., Inc., New York, 1969.

[37] J.-L. Lions And E. Magenes, Non-homogeneous boundary value problems and applications. Vol. I, Springer-Verlag, New York-Heidelberg, 1972. Translated from the French by P. Kenneth, Die Grundlehren der mathematischen Wissenschaften, Band 181.

[38] R. Rischette, T. N. Baranger, and N. Debit, Numerical analysis of an energy-like minimization method to solve the Cauchy problem with noisy data, J. Comput. Appl. Math., 235 (2011), https://doi.org/10.1016/j.cam.2010.12.019, https://hal.archives-ouvertes. fr/hal-00584553v3. 